\title{
NEW COMBINATIONS, RANK CHANGES, AND NOMENCLATURAL AND TAXONOMIC COMMENTS IN THE VASCULAR FLORA OF THE SOUTHEASTERN UNITED STATES. IV
}

\section{${ }^{1}$ Alan S. Weakley, ${ }^{2}$ Bruce A. Sorrie, ${ }^{3}$ Richard J. LeBlond, ${ }^{4}$ Derick B. Poindexter}

UNC Herbarium (NCU), North Carolina Botanical Garden, Campus Box 3280,

University of North Carolina at Chapel Hill, Chapel Hill, North Carolina 27599-3280, U.S.A. weakley@unc.edu, basorrie@gmail.com,richardleblond@charter.net,dbpoindexter@gmail.com

${ }^{5}$ Aaron J. Floden

Missouri Botanical Garden (MO) 4344 Shaw Blvd.

Saint Louis, Missouri 63110, U.S.A.

afloden@mobot.org

${ }^{7}$ Alan R. Franck

Dept. of Biological Sciences, OE 167

Florida International University, 11200 SW 8th St.

Miami, Florida 33199, U.S.A.

afranck@fiu.edu
${ }^{6}$ Edward E. Schilling

Dept. of Ecology \& Evolutionary Biology (TENN)

University of Tennessee

Knoxville, Tennessee 37996 U.S.A. eschilling@utk.edu

${ }^{8}$ John C. Kees

St. Andrew's Episcopal School 370 Old Agency Road

Ridgeland, Mississippi 39157, U.S.A.

johnckees@gmail.com

ABSTRACT

As part of ongoing efforts to understand and document the flora of the southeastern United States, we propose a number of taxonomic changes and report a distributional record. In Rhynchospora (Cyperaceae), we elevate the well-marked R. glomerata var. angusta to species rank. In Dryopteris (Dryopteridaceae), we report a state distributional record for Mississippi for D. celsa, filling a range gap. In Oenothera (Onagraceae), we continue the reassessment of the Oenothera fruticosa complex and elevate O. fruticosa var. unguiculata to species rank. In Eragrostis (Poaceae), we address typification issues. In the Trilliaceae, Trillium undulatum is transferred to Trillidium, providing a better correlation of taxonomy with our current phylogenetic understanding of the family.

\section{RESUMEN}

Como parte de los esfuerzos en marcha para comprender y documentar la flora del sureste de los Estados Unidos, proponemos un número de cambios taxonómicos y aportamos un registro de distribución. En Rhynchospora (Cyperaceae), elevamos la bien marcada R. glomerata var. angusta al rango específico. En Dryopteris (Dryopteridaceae), aportamos las citaciones a nivel estatal para Mississippi de D. celsa, llenando un hueco en su distribución. En Oenothera (Onagraceae), continuamos la revaloración del complejo Oenothera fruticosa y elevamos O. fruticosa var. unguiculata al rango de especie. En Eragrostis (Poaceae), tratamos de las tipificaciones. En Trilliaceae, Trillium undulatum se transfiere a Trillidium, aportando una correlación mayor entre la taxonomía y nuestro actual conocimiento de filogenético de la familia.

\section{INTRODUCTION}

As part of ongoing work on the Flora of the Southern and Mid-Atlantic States (Weakley 2015; Weakley 2018), as well as for general floristic, conservation, and scientific work in eastern North America, it is necessary or desirable to document taxonomic and nomenclatural changes and significant distribution records. In some cases, new combinations are needed to accurately reflect current taxonomic understanding. Some of these are rank changes, whereas others are generic transfers to apply new (or old) generic concepts to taxa that do not have corresponding available names at the specific or infraspecific level. We have also addressed various nomenclatural issues and clarified characters and identification of difficult groups in the regional flora.

We here present a fourth volume of such changes, contributed by eight authors. Primary authorship of the sections in this paper is as follows (and is also indicated at the beginning of each section): Rhynchospora (BAS, RJL, \& ASW), Dryopteris (JCK and ASW), Oenothera (BAS, RJL, \& ASW), Eragrostis (ARF), and Trillidium (AJF \& EES). 


\section{CYPERACEAE}

\section{Rhynchospora: A reassessment of R. glomerata var. angusta}

Primary authors: Bruce A. Sorrie, Richard J. LeBlond, and Alan S. Weakley

In her monograph of North American and West Indian Rhynchospora sect. Eurhynchospora, Shirley Gale named R. glomerata var. angusta Gale as distinct from the typical R. glomerata (L.) Vahl (Gale 1944). She stated "The achene of var. angusta is, as the name implies, narrower, somewhat shorter, with a slender accentuated gynophore as in R. microcephala." In contrast, the achene of the nominate variety "...is broadly ovoid with a short thick gynophore." Gale gave measurements: for var. angusta, achene width 1-1.1 mm, achene length $1.5-1.6 \mathrm{~mm}$, and tubercle length $1.3-1.8 \mathrm{~mm}$; for var. glomerata, achene width $1.2-1.4 \mathrm{~mm}$, achene length $1.5-1.7 \mathrm{~mm}$, but no data for tubercle length. Gale stated "The achene is the most important single character in the determination of a specimen, for, almost without exception, that of every species is distinct."

Gale described the range of var. angusta as Arkansas, western Louisiana, and eastern Texas, all west of the Mississippi River. She described the range of var. glomerata as the Coastal Plain from Delaware to Florida and Texas, inland to the Appalachians of the Carolinas, scattered in Tennessee, and in the drainages of the Arkansas, Red, and Sabine Rivers.

Despite Gale's careful monographic work, most authors of floras and floristic atlases since her monograph have not recognized var. angusta: Correll and Johnston (1970), Godfrey and Wooten (1979), Smith (1988), Thomas and Allen (1993), Taylor and Taylor (1994), Kral (2002), Diggs et al. (2006), and Gentry et al. (2013) all treat $R$. glomerata as a species with no infraspecific taxa. None of these authors provided an explanation for differing from her monographic work, nor did they provide a commentary, new data, or a stated rationale for adopting a new taxonomic concept (of recognizing one taxon rather than two). This pattern of not providing a basis for choosing not to recognize infraspecific taxa is discussed briefly by Weakley et al. (2017). McMillan (2007) included R. glomerata var. glomerata, implying recognition of var. angusta, but he omitted the latter from his treatment although it occurs in his area of coverage in Arkansas and Louisiana. Likewise, LeBlond (2015) included R. glomerata var. glomerata and mentioned var. angusta, but he did not provide a separate treatment as it does not occur within his area of coverage. Naczi and Moyer (2017) addressed the status of var. angusta (though somewhat obliquely), observing that "the holotype and one paratype of $R$. glomerata var. angusta fall within the continuum of R. glomerata (Fig. 3)." Thus, we are unaware of any publication since Gale (1944) that has given full recognition to var. angusta. Moreover, no one, including Gale (1944), has illustrated this taxon.

In order to re-evaluate whether var. angusta warrants recognition, and if so, at what rank, we borrowed specimens from BRIT, LL, LSU, NCU, OKL, SMU, TEX, UARK, and VDB; additional specimens from DUKE, FSU, MICH, and US were examined during prior investigations. Lists of specimens examined are found below. We focused measuring and comparing (non-statistically) the features previously used by Gale to recognize var. angusta — the same features that have proven most diagnostic in general in Rhynchospora taxonomy-achene body width and length and achene beak length. We measured these characters on specimens of both taxa, and of related species R. capitellata (Michx.) Vahl and R. leptocarpa (Chapm. ex Britt.) Small. Gale's measurements of achene length were shorter than ours—we judge that she omitted the gynophore but did not explicitly say so. Since the gynophore tapers into the achene body, it is difficult to detect a definite point of departure. Therefore, to provide more consistent measurements, we included the gynophore and measured the entire body from base of gynophore to base of tubercle.

Achene body width and tubercle length show a clear difference between the two taxa (Table 1). Achene body length shows less consistent difference, so we do not include it here as a key character to distinguish them, though the difference in achene body width combined with very similar lengths means that the length:width ratio and therefore overall shape differ noticeably. Images of achenes show a subtle but distinctive difference in shape and size between var. glomerata and var. angusta, comparable to that used to differentiate many Rhynchospora species (Fig. 1). The narrower body of var. angusta tapers more gradually to the gynophore than that of var. glomerata, which tapers rather abruptly, yet still exhibits a distinctly concave curvature, unlike 
TABLE 1. Measurements of achenes (average and range in $\mathrm{mm}$ ) of Rhynchospora glomerata var. glomerata and var. angusta. Body length includes the gynophore. $\mathrm{N}=27$.

\begin{tabular}{lll}
\hline & var. glomerata & var. angusta \\
\hline body width & $1.35(1.2-1.5)$ & $1.0(0.9-1.2)$ \\
body length & $2.0(1.9-2.1)$ & $1.9(1.7-2.0)$ \\
tubercle length & $1.7(1.5-1.9)$ & $1.45(1.1-1.7)$ \\
\hline
\end{tabular}

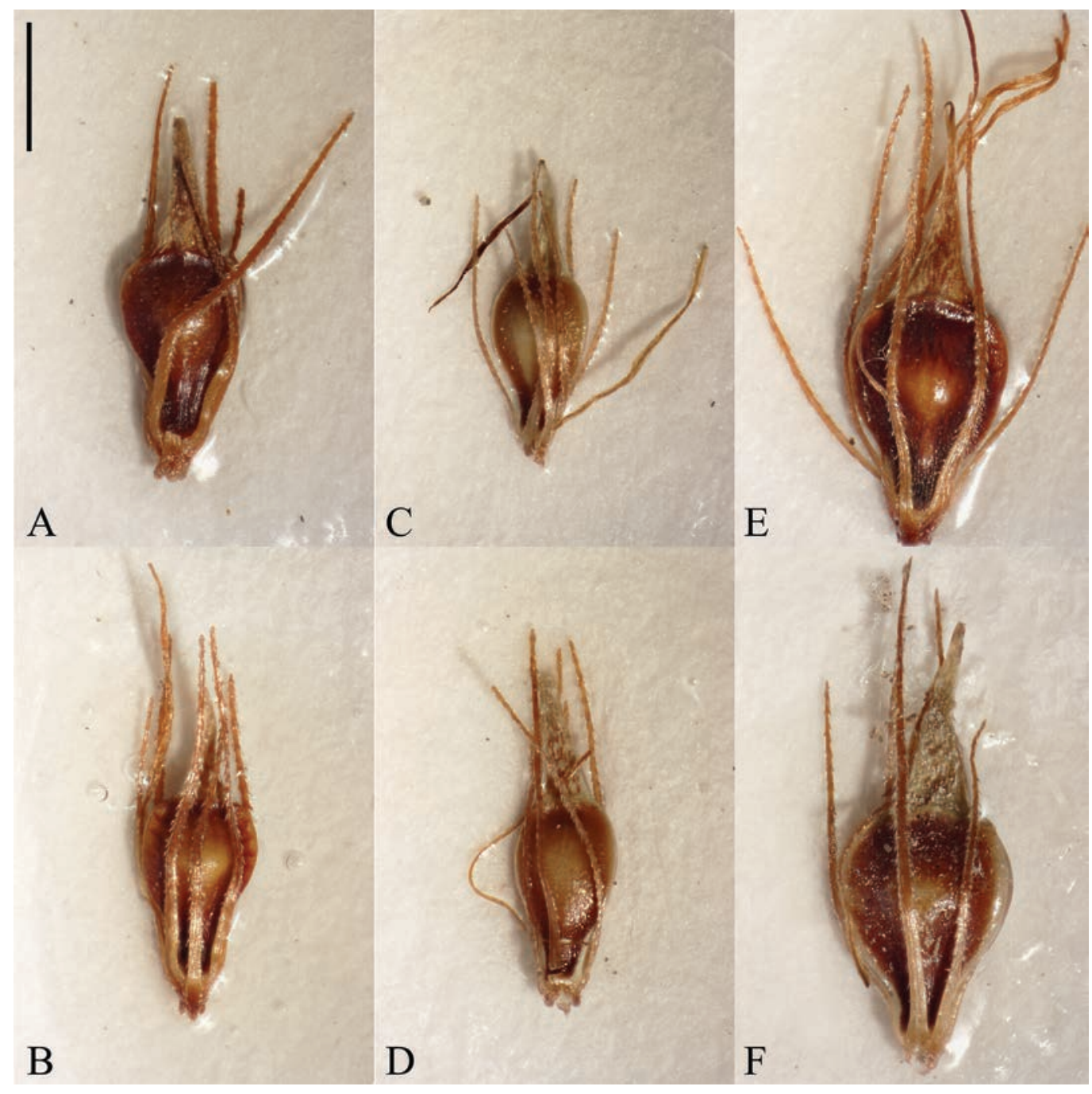

FIG. 1. Achenes of Rhynchospora glomerata var. angusta (A-B), R. capitellata (C-D), and R. glomerata var. glomerata (E-F). Scale bar $=1 \mathrm{~mm}$. A and $B$ are from Arkansas and Texas, respectively; $C$ and D are from Texas and North Carolina, respectively; $E$ and $F$ are from Oklahoma and North Carolina, respectively. Photos by D.B. Poindexter. 
R. capitellata [compare Naczi \& Moyer's Fig. 5 (2017)]. Mean achene body length of var. angusta (1.9 mm) is much closer to var. glomerata (2.0) than to R. capitellata (1.6) or R. leptocarpa (1.7). Blake's (1918) illustrations of the achenes of R. glomerata, R. capitellata, and R. capitellata var. leptocarpa mirror those of Naczi and Moyer's; he did not recognize infraspecific taxa within our modern circumscription of $R$. glomerata.

An additional character, perhaps useful in the field as well as in the herbarium, is the usually greater number of spikelets in each cluster of var. angusta, which gives them a more rounded appearance in most (but not all) specimens (Figs. 2, 3). Finally, we detected only five equivocal specimens among the hundreds of specimens examined-a very small percentage—which suggests that the two taxa interact very little despite a large geographical area of overlap (see below).

Regarding Naczi and Moyer's (2017) decision to merge var. angusta within nominate R. glomerata based on a principal components analysis, we note that in their Figure 3 the symbol for var. angusta falls at the edge of the scatter plot, suggesting that additional study may be warranted using a larger sample size. Our study of hundreds of specimens each of var. glomerata and var. angusta definitively separates the two.

In considering the appropriate taxonomic treatment (rank) of var. glomerata and var. angusta, we note the following points:

1. Several different quantitative features of the achene and its tubercle (the most taxonomically revealing feature in the genus) differ in a correlated manner between the taxa and serve to reliably distinguish nearly all specimens.

2. Another character that we did not quantify (infructescence cluster shape, probably based on number of spikelets in each cluster) is also correlated with the spikelet and tubercle size and shape differences (it also aligns by taxon).

3. When specimens are mapped geographically based on their identification by these features, two discrete distributions result, with var. glomerata having a wide distribution in the SE US and var. angusta restricted to the West Gulf Coastal Plain (west of the Mississippi River) (Figs. 4, 5). No specimens of var. angusta have been found east of the Mississippi River, suggesting that the taxon has a discrete range. Both of these are very common distribution patterns, each with hundreds of other plant species with very similar distributions (Sorrie \& Weakley 2001); thus they are both very plausible distributions for plant species.

4. The distributions of the taxa largely overlap west of the Mississippi River, and the two occur in a similar range of habitats and are likely syntopic (together at the same sites). Yet only a few aberrant collections have been seen that might represent hybridization or introgression (or something else, like developmental aberrancies or hybridization or introgression of one or both with a third species).

Based on all evidence available (measurements of achene and tubercle characters, other less quantifiable morphological characters, and the geographic pattern of the the two entities) we propose that the most reasonable, parsimonious, and conservative (in several senses of the word) way to treat these two taxa is as independently evolving evolutionary lineages-i.e., species. Therefore, we elevate $R$. glomerata var. angusta to species rank.

Rhynchospora angusta (Gale) Sorrie, LeBlond, \& Weakley, comb. et stat. nov. BAsionym: Rhynchospora glomerata (L.) Vahl var. angusta Gale, Rhodora 46(544):114-115. 1944. Type: TEXAS. WALlER Co.: brook banks, Hempstead, Jun 101872, Hall 718 (HOLOTYPE: GH; ISOTYPES: MO, US).

The following key effectively separates R. glomerata and R. angusta.

1. Achene body 0.9-1.2 (mean 1.0) mm wide; achene tubercle 1.1-1.7 (mean 1.45) mm long

1. Achene body 1.2-1.5 (mean 1.35) mm wide; achene tubercle 1.5-1.9 (mean 1.7) mm long

R. angusta R. glomerata

Additional, more statistical analyses would be desirable, and we plan to conduct them and report on the results.

Specimens examined determined as Rhynchospora angusta.

ARKANSAS. Ashley Co.: Airport Prairie 5 mi NE of Crossett, drainage slough, 27 Jun 1986, Sundell et al. 7183 (BRIT, VDB). Bradley Co.: Warren, low wet areas, 3 Jul 1943, Demaree 24359 (NCU, VDB); Warren Prairie, marshy prairie, 4 Jul 1982, Sundell \& McCurley 2757 (LSU, UARK, VDB); same place, 20 Jun 1982, Sundell $\&$ McCurley 2703 (VDB). Calhoun Co.: Hampton vicinity, 10.2 mi S of US 167, roadside ditch, 13 Sep 2002, Hyatt 11267 (UARK); edge of "Lost 40," old-growth loblolly pine/hardwood forest, 18 Jul 2000, Witsell 00-143 (UARK); low cutover woods $2 \mathrm{mi}$ SW of Harrell, 27 Aug 1987, Sundell \& Etheridge 7828 (LSU); sandy gravelly clay $4.5 \mathrm{mi}$ S of Hampton in oak-pine flats, $14 \mathrm{Jul}$ 1981, Kral 67516 (VDB); clay of pine-oak flats $10 \mathrm{mi}$ S of Hampton, Kral 24532 (VDB); Artesian quadrangle, N of gravel road just W of 


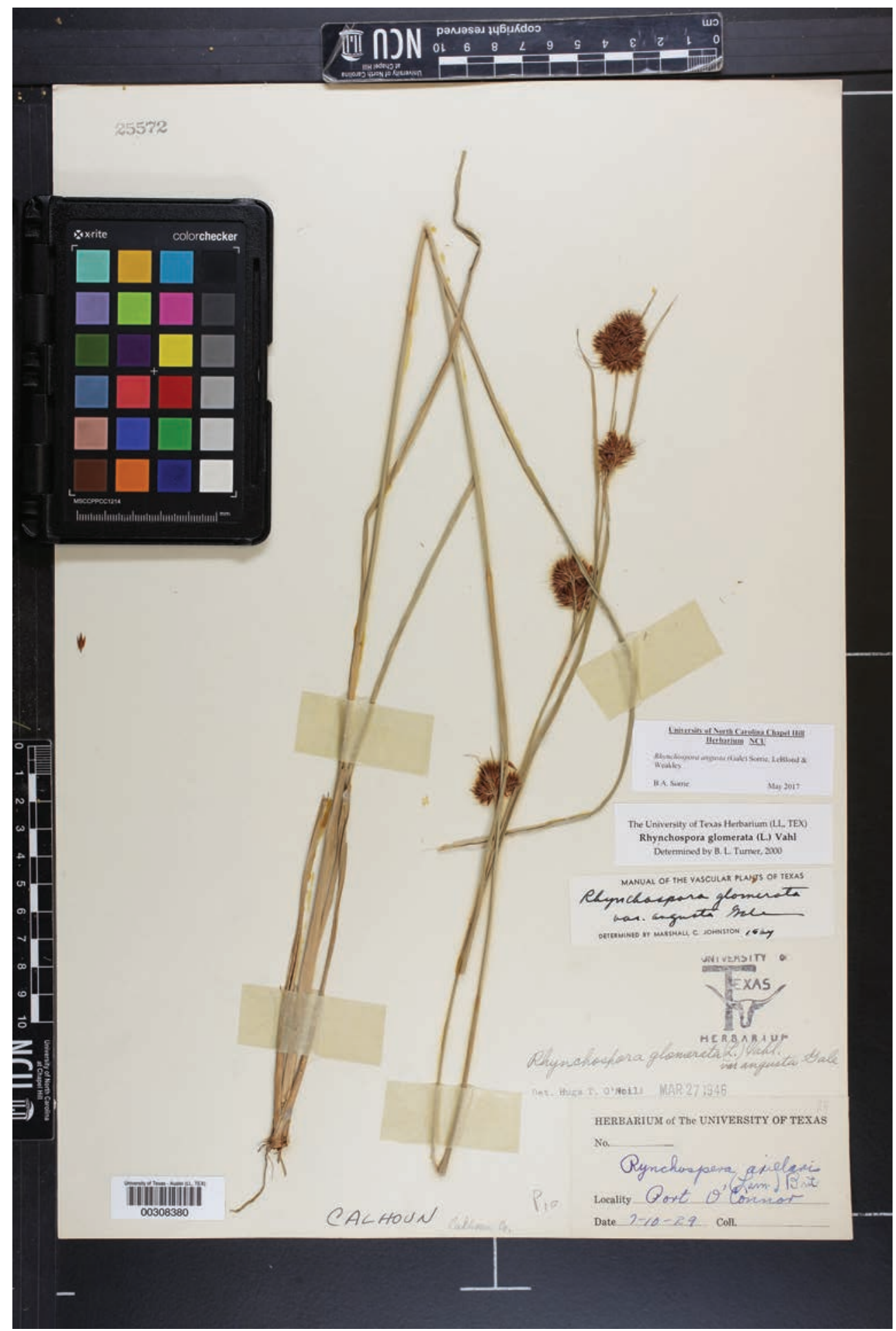

FIG. 2. Specimen of Rhynchospora angusta from Texas. Note rounded spikelet clusters and annotation in Gale's hand. 


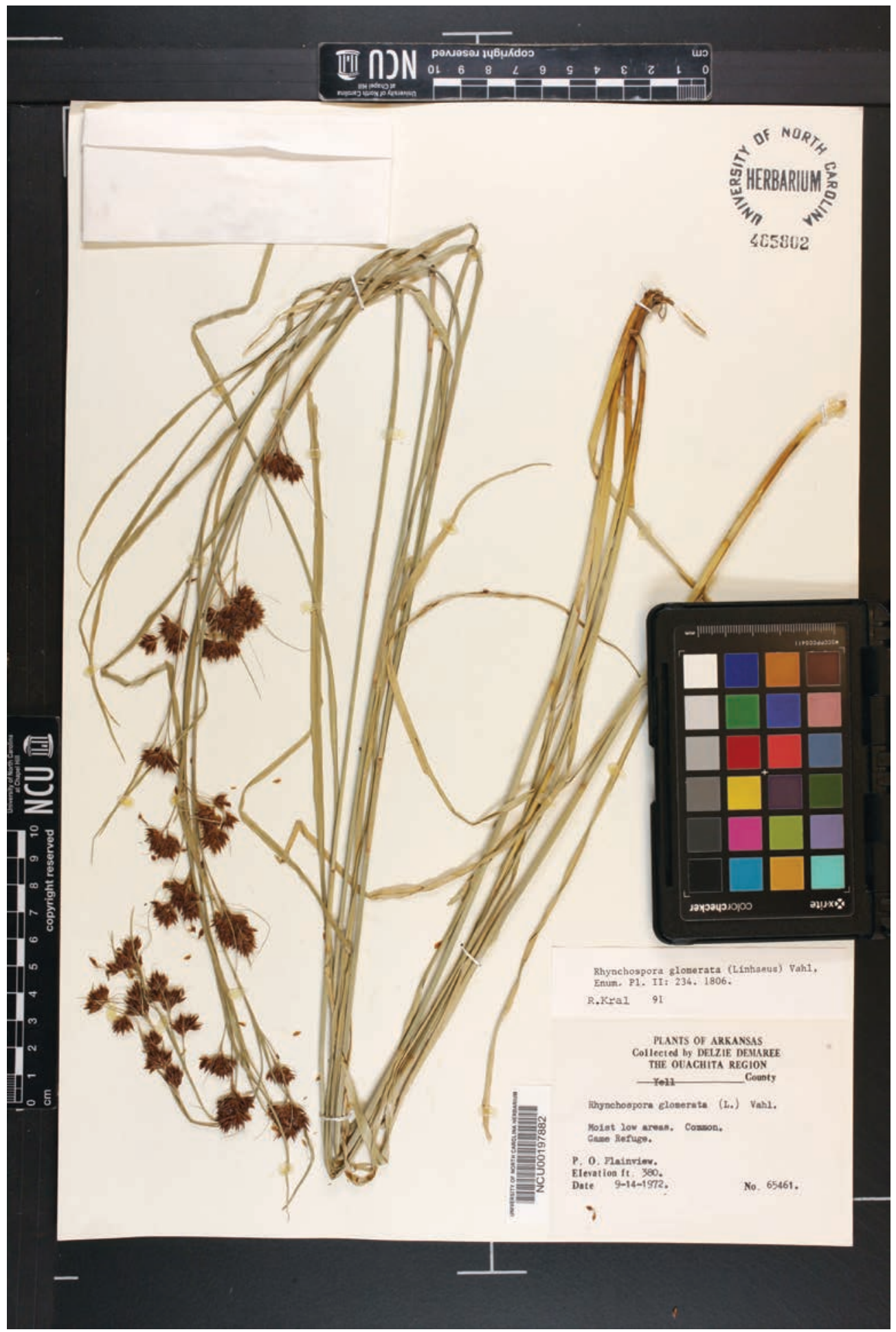

FIG. 3. Specimen of Rhynchospora glomerata from Arkansas. 


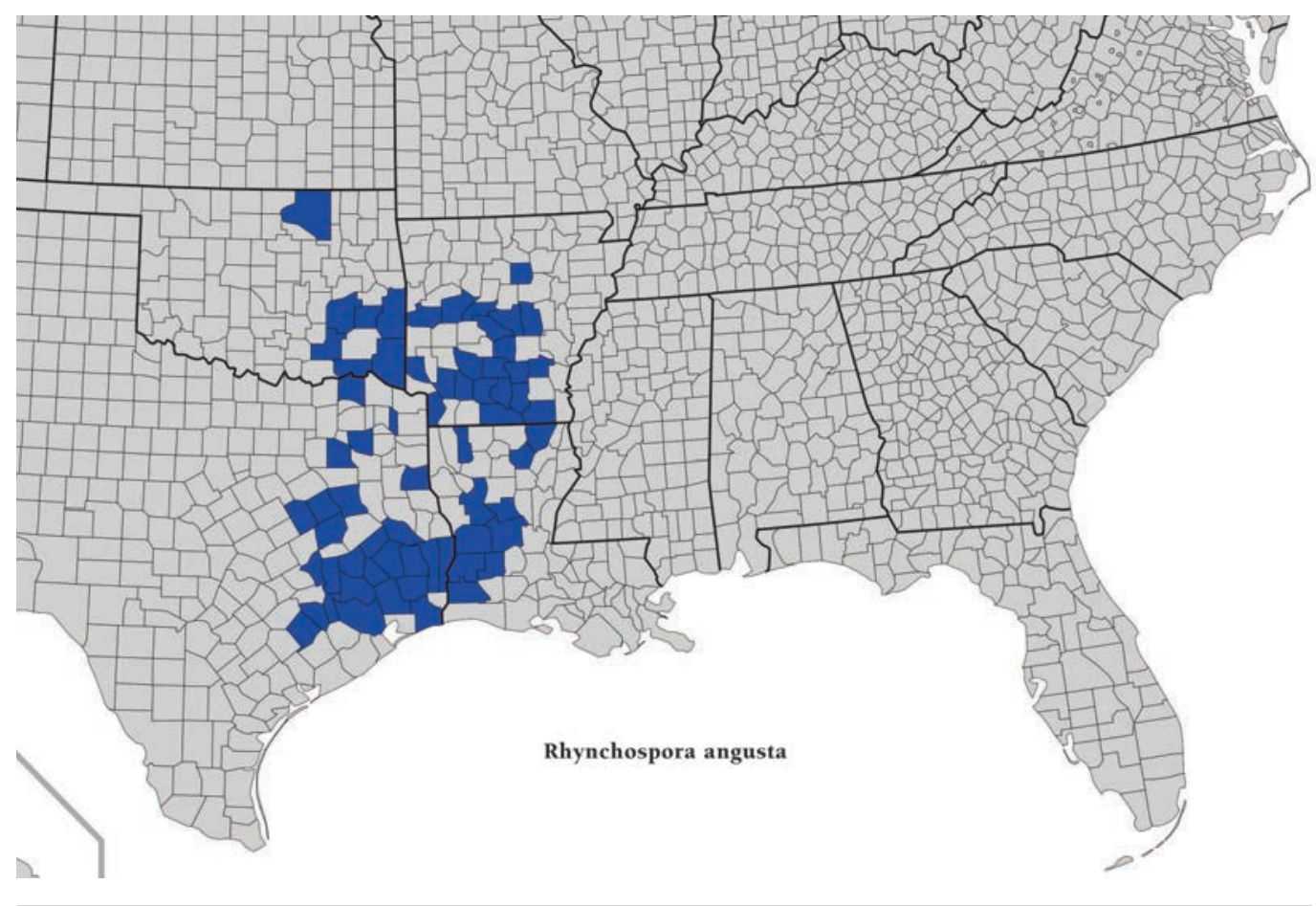

FIG. 4. Distribution map of Rhynchospora angusta. Based entirely on specimens examined by the authors.

abandoned railroad, wet cutover Pinus taeda flatwoods, 14 Jun 1985, Orzell \& Bridges 2119 (BRIT). Clark Co.: low, poorly drained ditches, 8 Jul 1967, Tucker 5748 (NCU); flat wet woods, Randolph Memorial, P.O. [Post Office] Whelen Springs, 28 Jul 1970, Demaree 62543 (VDB); sandy creek bottom. Cleburne Co.: pond margin, old stone quarry, 24 Jul 1968, Demaree 58696 (NCU, VDB). Cleveland Co.: Mill Creek Geocarpon site, 29 Jun 1985, Shepherd \& Schiffl 243 (UARK); 6 mi NE of Rison, 0.8 mi W on Dunkeffie Road, 17 Aug 2005, McElderry \& Williams 2892 (UARK); right-of-way and woods at Ark. 114 bridge on Big Creek, 18 Sep 1999, Thomas \& Sundell 163,045 (VDB). Dallas Co.: pond margins, P.O. Fordyce, 20 Jul 1939, Demaree 19520 (SMU); Princeton vicinity, East Tulip Creek, sandbar, 14 Sep 2002, Hyatt 11288 (UARK); Sparkman Road 1/2 mi W of Sparkman, clearcut bordered by marshy area, 28 Jul 2004, Nunn \& Jacobs 10102 (UARK). Grant Co.: 4 mi W of Sheridan, $4.8 \mathrm{mi} \mathrm{N}$ on US 270, roadside ditch, 16 Jul 2005, McElderry $\&$ Williams 2454 (UARK). Hempstead Co.: low wet bottom, P.O. McCaskill, 18 Aug 1966, Demaree 54102 (NCU, VDB); railroad right-of-way, 8 Jul 1967, Tucker 5778 (NCU); along hwy. 32, 2 Sep 1977, Redfearn et al. 31415 (NCU). Lincoln Co.: 9.5 mi NW of Star City, 5.8 mi N of SH 212 on CR 10, 16 Aug 2005, McElderry et al. 2824 (UARK); $4.9 \mathrm{mi}$ W of Star City, $0.3 \mathrm{mi}$ E of SH 11, 16 Aug 2005, McElderry et al. 2776 (UARK). Lonoke Co.: rice area, common, P.O. Carlisle, 10 Jul 1973, Demaree 67097 (VDB). Miller Co.: wet ditch W of Ark. 237, SW of Ferguson, 23 Sep 2000, Reid E Gentry 1452 (UARK); Three States, at La. state line, 17 Jul 1999, Hyatt 8696 (UARK). Nevada Co.: roadside just W of Dorcheat Bayou Bridge, 23 Jun 2002, Marsico \& Clifton 2841 (UARK). Ouachita Co.: $1.4 \mathrm{mi}$ E of county road 67 on county road 70, oil drilling operation, 8 Sep 2000, Doffitt 997 (VDB). Perry Co.: Ouachita NF, South Fourche La Fave River at low-water bridge, 4 Aug 2002, Nunn 6861 (UARK). Pulaski Co.: Camp Robinson, in drainage, 31 Jul 1942, Tolstead 5648 (NCSC, SMU); 11 mi S of Little Rock, 145th street exit off US 65, gas line, 12 Aug 1992, Bryson 12058 (VDB); Grand Prairie, Jul, Harvey 7 (GH, MO cited by Gale). Saline Co.: Bauxite, Sep 1947, Moore 470484 (UARK); W on US 165, 0.5 mi from US 65, open low wet area along edge of pine woods, 12 Aug 1992, Bryson 12061 (VDB). Scott Co.: Ouachita NF, small stream crossing FS road 776A, 1 mi E of 776, 31 Aug 1980, Barber 2270 (UARK). Sevier Co.: West Otis, 26 Jul 1937, Brinkley 388 (TEX). Union Co.: damp sand roadside, Champagnolle Road, vicinity of El Dorado, 4 Aug 1990, Sadler 485 (UARK). Yell Co.: Ouachita NF, FS Road 17 at Irons Fork Creek, nearly dry bouldery streambed, 24 Sep 1990, Bates 10425 (UARK, VDB). LOUISIANA. Allen Par.: borrow pit 5 mi E of Sugartown, 20 Jun 1968, Thieret 29552 (DUKE); wet roadside ditch of rte. 26, $11.2 \mathrm{mi} \mathrm{SE}$ of Oberlin, 28 Jun 1981, Allen 10964 (LSU); pine flatwoods 8 mi E of Oberlin, 11 Jun 1982, Allen 12241 (LSU); 4 mi N of Oakdale on US 165, railroad right-of-way, 22 Aug 1980, Carter 2485 (VDB); roadside clearing on US 165, 1.3 mi N of Jefferson Davis Parish, 23 Jun 1982, Kessler 6305 (BRIT). Beauregard Par.: wet longleaf savannah with pimple mounds, 14 Jul 1991, Orzell \& Bridges 17290 (MICH); roadside clearing on La. 27, 10.5 mi S of La. 12, 23 Jun 1982, Kessler 6395 (VDB); W side of De Ridder on heavy clay of pond bank, 17 Jul 1964, Kral 20803 (VDB). Calcasieu Par.: low prairies, common, Aug-Sep 1898, Mackenzie 442 (MO, NCU, NY); Lake Charles, 7 Aug 1897, Tracy s.n. (GH, US); common in wet places in prairies S of Sulphur, 17 Jul 1935, Brown 5903 (LSU); 


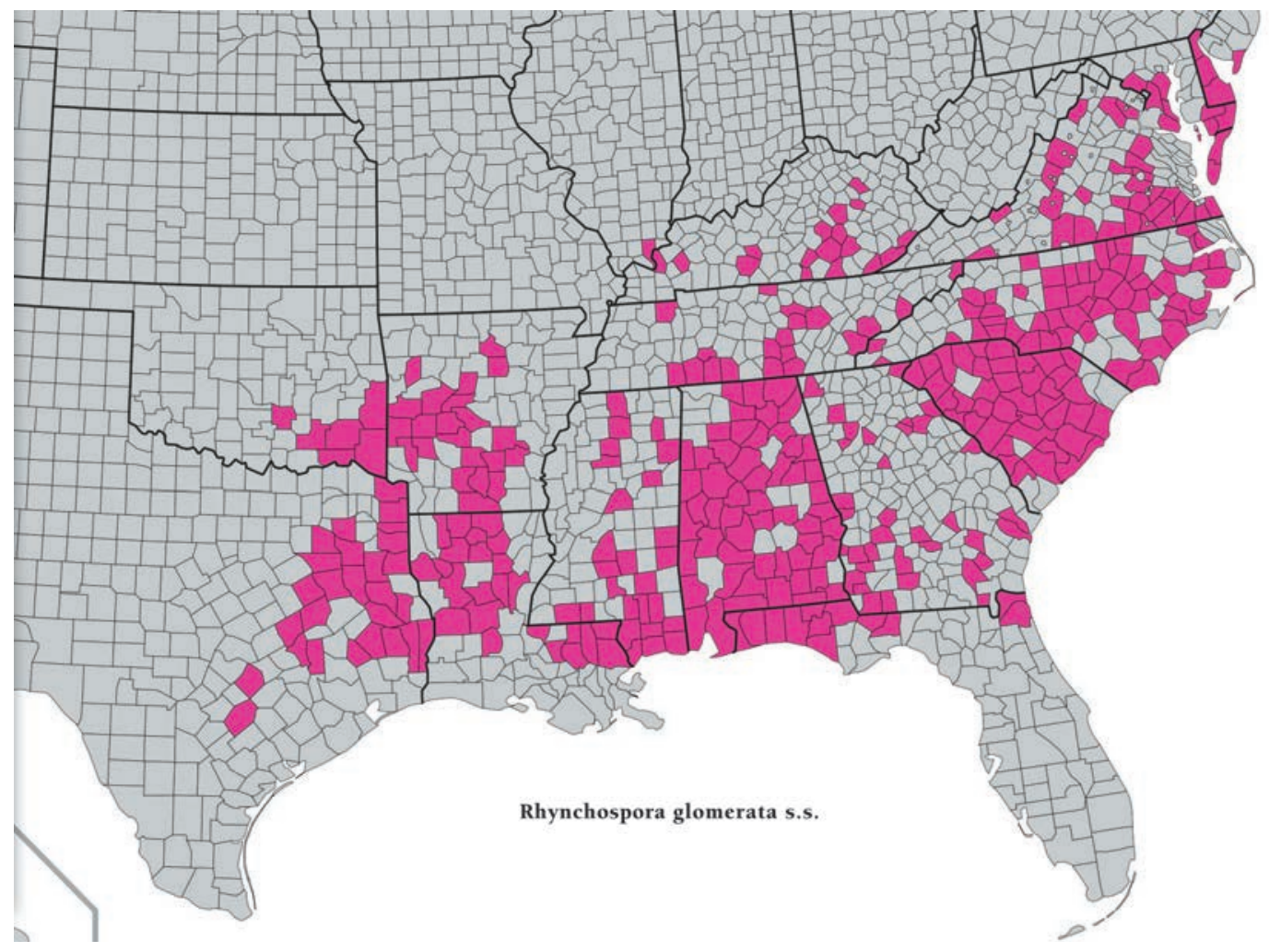

FIG. 5. Distribution map of Rhynchospora glomerata. Records from west of the Mississippi River are based on specimens examined by the authors; records from east of the river are from Kartesz (1998).

mixed pine-hardwoods W of Sulphur, 19 Oct 1940, Brown 8593 (LSU); Sweet Lake Prairie, 2 mi S of Holmwood, 21 Jun 2010, Reid \& Bass 7469 (LSU); no location, 13 Jul 1999, Hill s.n. (BRIT). Grant Par.: 4.3 mi S of Georgetown, 6 Sep 1965, Thieret 20247 (US); Thomas 2999 (NCU); roadside ditch in longleaf pine hills near Pollack, 29 Jul 1935, Brown 5977 (LSU). Morehouse Par.: 7 mi NW of Bastrop, roadside ditch, 1 Jul 1968, Thieret 29877 (DUKE); pinewoods 1 mi E of La. 139, 1 mi N of Beckman Fire Tower, 27 Sep 1969, Thomas 16783 (VDB). Natchitoches Par.: Kisatchie NF, Kisatchie District, Bayou Campground, common on streambank, 9 Sep 2002, Hyatt 11230 (LSU); Kisatchie NF, Winn District, Dragonfly Bog, 11 Sep 2001, Hyatt 10576 (VDB); Kisatchie NF, Winn District, Packton vicinity, wet mudhole in old logging landing, 14 Aug 2001, Hyatt 10467 (VDB); Kisatchie NF, Penrod pitcher plant bog, 11 Aug 1990, MacRoberts \& MacRoberts 1117 (VDB). Ouachita Par.: 3 mi W of Calhoun, 21 Jul 1969, Thieret 31759 (DUKE). Rapides Par.: drying pond at roadside, E of Flatwoods, 11 Jul 1968, Thieret 30335 (DUKE, FSU, SMU); ditch in Holloway Prairie, 24 Jun 1936, Brown 6442 (LSU); pinelands near Alexandria, Jul 1906, Cocks s.n. (LSU); open piney woods in vicinity of Indian Creek Rec. Area, 9 Aug 1996, Lasseigne 10946 (LSU). Vernon Par.: roadside ditch 9 mi N of Merryville, 8 Jul 1968, Thieret 30152 (DUKE, FSU); near Dowden Creek, 5 mi E of Hornbeck, Thieret 26922 (DUKE, FSU); Kisatchie NF, Vernon District, Cooter's Bog, 30 Jul 2002, Ferguson \& Karamen-Castro 680 (LSU); Fort Polk, Texas Ave. at Mill Creek Road, 17 Jun 2002, Allen \& Chance 18933 (VDB). Webster Par.: margin of pond near Minden, 15 Jul 1934, Brown 5354 (LSU). No parish: "Louisiana," no date or collector, LSU \#29082. OKLAHOMA. Atoka Co.: abundant, roadside ditch 5 mi SE of Atoka, 19 Jul 1969, Massey \& Massey 2494 (NCU, OKL); rocky stream, Crooked Creek, 27 Jul 1979, Taylor 27831 (BRIT). Choctaw Co.: hillside bog 4 mi NE of Swink, 18 Oct 1974 , Taylor 17165 (OKL). Latimer Co.: $8 \mathrm{mi}$ W and $1.5 \mathrm{mi}$ S of Talihins, 9 Aug 1948, Waterfall 8549 (OKL, TEX). Le Flore Co.: Ouachita NF, 5.7 mi E of Big Cedar, 11 Aug 1996, Magrath \& Carpenter 19542 (OKL, TEX); Ouachita NF, Homer Johnston WMA, 15 Aug 1991, Magrath 18413 (OKL); Cucumber Creek Preserve, oak-hickory forest, 20 Jul 2005, Hoagland \& Crawford CC275 (OKL); Cedar lake, buttonball zone, 9 Jul 1949, Penfound P298 (OKL). McCurtain Co.: lower Mountain Fork River, cobble bar, 24 Jul 2012, Buthold \& Hoagland AB10080 (OKL); Broken Bow Lake, near stream, 16 Jul 2009, Buthold \& Hoagland AB8252 (OKL); edge of water, Yanubbee Creek, 2.5 mi N of Broken Bow, 12 Sep 1966, Crutchfield 2193 (LL). Osage Co.: seep/spring at Western Wall Unit of Osage WMA, 6 Sep 2011, Hoagland E Ewing s.n. (OKL). Pittsburg Co.: sluggish stream on hwy. 43, 3 mi E of Stringtown, 23 Jul 1967, Mitchell 3410 (LL). TEXAS. Anderson Co.: Gus Engling WMA, Beaver Pond \#9, 25 Jul 1986, Hogan 049 (BRIT); Gus Engling WMA, 6 mi NW of Tennessee Colony, Marsh, Jr. 200 (TEX); Beaver Creek, 
grass-Alnus-Myrica bog, 14 Jul 1990, Mayfield 580 (BRIT). Angelina Co.: edge of pond in open pine woods near Bouton Lake, Angelina NF, 12 Jul 1958, Correll \& Johnston 19659 (FSU, LL, NCU); pond on hwy. 69, 1 mi NE of Huntington, 27 Aug 1967, Mitchell 3996 (LL). Austin Co.: prairie, Wallis, 3 Jul 1939, Tharp s.n. (SMU, TEX, VDB); 1 mi E of Bellville, seepage bog, 25 Jul 1964, Kral 21070 (VDB). Brazos Co.: post oak savanna, E of Hwy. 6, 23 Oct 1981, Kessler 5161 (BRIT); Minter Springs, Wellborn, 10 Jun 1963, Massey 198 (LL); same place, 15 Jun 1965 , Massey 968 (SMU); seepage areas of post oak woodlands above Minter Spring, 6 Jun 1989, Orzell \& Bridges 10367 (TEX). Calhoun Co.: Port O'Connor, 20 Jul 1929, no collector s.n. (TEX). Colorado Co.: Eagle Lake, 11 Jul 1929, Tharp s.n. (TEX); sandbars of San Bernard River, 9.8 mi W of Sealy, 27 Sep 1988, Orzell \& Bridges 8603 (TEX); Attwater Prairie Chicken NWR, weedy margins of lake, 28 Jul 2012, Rosen et al. s.n. (TEX). Freestone Co.: 12 2/3 mi SE of Fairfield, moist soil of creek branch, 2 Nov 1947, Cory 53918 (FSU, SMU, US). Grimes Co.: hardwood forest 2.9 mi E of Carlos on hwy. 30, 9 Aug 1989, Ajilvsgi s.n. (BRIT). Hardin Co.: Big Thicket, 20 Jul 1929, Tharp s.n. (TEX); S of Village Creek, 28 Jun 1952, Tharp \& Tyson s.n. (NCU, SMU); $7.4 \mathrm{mi} \mathrm{NW}$ of Silsbee, then 2/3 mi S, moist pine woods, 9 Jul 1949, Cory 56649 (SMU); $3 \mathrm{mi}$ N by W of Votaw, borrow ditch, 9 Jul 1949, Cory 56674 (SMU); 2.6 mi E of Camp Jackson, 13 Sep 1936, Cory 19711 (GH, USNC, not seen, cited by Gale); swamp forest near Ariola, 18 Aug 1942, Lundell \& Lundell 11515 (MICH, SMU); roadside clearing on hwy. 770, $1.6 \mathrm{mi}$ S of hwy. 326, 23 Jun 1982, Kessler 6251 (SMU); Lance Rosier Unit, Big Thicket National Preserve, hummocky wet land, 7 Sep 1983, Watson 3402, -03, -04 (all SMU); Lance Rosier Unit, Big Thicket National Preserve, wet pine savanna, 18 Sep 1997, MacRoberts \& MacRoberts 3646 (TEX); roadside ditch with water, 13 Nov 1945, Cory 50788 (MICH); NW of Kountze, 4 Jul 1992, Jones \& Jones 9134 (MICH); Village Creek SP, remnant longleaf pine wet savanna, 23 Oct 2003, Sanders 6319 (BRIT, TEX); just NW of Saratoga, artificial pineland pond, 25 Jul 1964, Kral 21085 (VDB). Harris Co.: Cypress City, Boll 866 (GH, not seen, cited by Gale); E side of road across from Westfield Cemetery, moist grassy meadow, some standing water, 14 Jun 1961, Traverse 2472 (LL, SMU); sandy soil, native prairie along Beltway 8, 28 Jul 1990, Brown 14730 (TEX). Jasper Co.: 3 mi S of Letney, pitcher plant bog associated with creek, 6 Aug 1976, Nixon et al. 7318 (BRIT); mesic-hydric area, edge of forest, 17 Jun 1989, Jones \& Jones 3160 (MICH); 1 mi E of Kirbyville, edge of pond between road and railroad, 30 Oct 1968, Correll \& Correll 36683 (LL); Evadale, 23 Aug 1941, Tharp s.n. (TEX); pond in Evadale, 31 Oct 1968, Correll \& Correll 36710 (LL); sandy pine woods along stream, 11 mi NW of Jasper, 12 Jul 1958, Correll \& Johnston 19619 (LL). Jefferson Co.: roadside, 27 Jun 1944, Higginbotham 30 (US); Across from Westfield Cemetery, moist grassy meadow, 14 Jun 1961, Traverse 2472 (BRIT). Lamar Co.: seepage and sandy stream on E edge of Direct, 20 Sep 1969, Correll 37933 (LL). Leon Co.: bog between Keechi Creek and Keechi, 1 Aug 1943, Barkley 13505 (GH, US); 1 2/3 mi NW of Buffalo, moist soil on roadside, 30 Jun 1948, Cory 54572 (LL, SMU). Liberty Co.: 21 mi SE of Cleveland, mixed pine-hardwoods foreat with wet depressions, 3 Jul 1950, Webster \& Wilbur 3186 (MICH, SMU, US); Tanner Bayou W of Capers Ridge, marsh area, 13 Oct 1972, Nixon et al. 5847 (BRIT); wet pasture behind business at jct. rte. 105 and rte. 321, W of Cleveland, 28 Jun 1997, Rosen 382 (LSU); Big Thicket, Loblolly Unit, E of county road 2071, 24 Jun 2007, Brown 32301 (BRIT). Limestone Co.: 4 mi SE of Donie, on hwy. 80 and E of hwy. 39 , margin of Lynn Creek, 8 Sep 1979, Lipscomb 3103 (SMU). Montgomery Co.: Conroe, 10 Jul 1929, Tharp s.n. (MICH); 3 mi NW of Conroe, sandy drainage way, edge of pine woods, 13 Oct 1953, Shinners 16598 (SMU, UARK); SE of Conroe, 19 Jun 1992, Jones 9053 (MICH); SE of W.E. Jones State Forest, 16 Jul 1992, Jones 9189 (MICH); Lake Houston State Park, around Cat Pond, 20 Aug 2003, Sanders 6061 (BRIT, TEX); Horsepen Branch, $0.25 \mathrm{mi} \mathrm{N}$ of Needham Road, bottomland hardwood forest, 16 Jul 1992, Jones \& Griffin 9189 (BRIT). Morris Co.: Daingerfield SP, moist woodland, 6 Sep 1947, Whitehouse 19165 (SMU); Daingerfield SP, trail along lake, 25 Jul 1976, Fleetwood 12346 (BRIT). Newton Co.: open hydric roadside, 27 Jun 1991, Jones \& Wipff 7229 (MICH); 21 mi N of Deweyville, 4 Oct 1934, Cory 10866 (GH, cited by Gale); off highway 87, 4 mi N of Highway 12, clearcut, 13 Jul 2007, Allen et al. 20381 (LSU, TEX); off highway 87, 8 mi S of Bleakwood, around oil well, 13 Jul 2007, Allen et al. 20415 (BRIT, LSU, TEX); same place and date, Allen et al. 20408 (LSU, TEX); same place, 2 Nov 2007, Allen et al. 20507 (LSU); off rte. 82 at State Forest, $5 \mathrm{mi}$ SW of Bleakwood, 25 Jun 2010, Allen \& Megyeri 21989 (LSU); 40 mi N of Orange, roadside ditch, 3 Oct 1964, Johnston 7160b (LL); wet savanna along route 87, 14 Sep 1967, Correll 36538 (LL); farm road 1416 E of Kirbyville, open hydric roadside, 27 Jun 1991, Jones \& Wipff 7229 (VDB). Panola Co.: county road 446 S of De Berry, small roadside pond in young pine plantation, 25 Jun 1991, Jones \& Wipff 7212 (VDB). Polk Co.: Big Sandy Creek at jct. with hwy. 1276, S of Dallardsville, dry stream bottom, 17 Jul 1958, Traverse 860 (LL, SMU); just W of Carmona at creek, 30 Jun 1963, Correll et al. 28091; 28092 (both LL); wet swale 7 mi E of Livingston, 18 Aug 1966, Correll \& Correll 33453 (LL). San Jacinto Co.: roadside clearing on hwy. 2025, 6.5 mi N of hwy. 59, 25 Jul 1981, Kessler 4706 (BRIT, LSU, VDB); shoulder of logging road through loblolly pine-oak forest, 4-5 mi N of Cleveland, 6 Jul 2009 , Carr 28186 (TEX); opening in loblolly pine-oak forest, 4-5 mi N of Cleveland, 7 Jul 2009, Carr 28213 (TEX). Trinity Co.: seepage swale in cutover longleaf pine savanna, 29 Jun 1989, Orzell \& Bridges 10873 (TEX); moist sand in fire-maintained pine-post oak savanna, Brushy Creek WMA, 6 Aug 1997, Carr 16797 (TEX). Tyler Co.: pitcher plant bog 16 mi S of Woodville, 4 Oct 1945, Cory 50057 (SMU); highway 69 N of Colmesneil, 10 Aug 1968, Correll \& Correll 36040 and 36046 (both LL); edge of stream, Clear Fork Creek, SW of Colmesneil, 18 Aug 1968, Correll \& Correll 33470 (LL); Big Thicket National Preserve, Lance Rosier Unit, wetland pine savanna, 8 Jul 1998, Carr 17710 and 17703-A (both TEX); boggy wetland, Big Thicket Bogs and Pineland Preserve, 25 Sep 1997, Carr 17086 (TEX); gulch formed by erosion, 6.5 mi E of Chester, 19 Oct 1967, Correll 35194 (LL). Van Zandt Co.: shaded margin of inlet of reservoir, Purtis Creek State Recreation Area, 3 Sep 1992, Carr 12291 (TEX). Walker Co.: Hunstville, 20 Jun 1935, Tharp 24288 (FSU, TEX, US). Waller Co.: brook banks, Hempstead, 10 Jun 1872 , Hall 718 (GH type; isotypes MO, US!). Wood Co.: 13 mi S of Quitman, borrow ditch above creek, 8 Aug 1950, Cory 57686 (SMU). County unknown, Harrisburg, 26 Jun 1875, Joor 1010 (MICH).

Specimens examined determined as Rhynchospora glomerata s.s. from west of the Mississippi River (dates and localities omitted to save space. Note that we examined hundreds of specimens from east of the Mississippi River and found none that matched var. angusta. All of the achenes displayed the broad body and long tubercle of var. glomerata; we have not listed them).

ARKANSAS. Bradley Co.: Leslie \& Clark 1112 (UARK); Demaree 19551 (SMU). Calhoun Co.: Demaree 23362 (SMU). Clark Co.: Demaree 58566 (VDB). Cleburne Co.: Demaree 42971 (SMU). Cleveland Co.: McElderry \& Williams 2890 (UARK). Columbia Co.: Demaree 62464 
(VDB). Conway Co.: Moore 420253 (UARK). Franklin Co.: Bryson 12051 (VDB). Garland Co.: Eggert s.n. (BRIT, NCU); King 201 (UARK); Hartsoe s.n. (UARK); Kral 90097; 90101 (both VDB); Demaree 11020 (SMU). Grant Co.: Bryson 12062 (VDB). Hot Spring Co.: Demaree 25662 (SMU). Howard Co.: Demaree 42738 (VDB). Jefferson Co.: Locke 2105 (UARK); Bryson 12065 (VDB). Lincoln Co.: McElderry et al. 2802 (UARK). Montgomery Co.: Uttal 10188 (SMU, VDB). Ouachita Co.: Demaree 48170 (NCU). Perry Co.: Demaree 10980 (SMU); Reid \& Marsico 2824 (UARK). Pike Co.: Hyatt 3574.55 (UARK). Polk Co.: Witsell \& Simon 01-0580 (UARK). Saline Co.: Tucker 6508 (NCU); Demaree 44732 (VDB); 65268 (NCU, VDB); Moore 470484 (UARK); Moore 32903 (UARK). Scott Co.: Morse 3900; 3868 (both VDB). Stone Co.: Beer 93-146 (UARK). Union Co.: Hoiberg 521 (SMU); Kral 24548 (VDB); Sundell \& McDonald 7656 (LSU). Yell Co.: Demaree 65461 (NCU, VDB); Nunn 6663 (UARK). LOUISIANA. Acadia Par.: Langlois s.n. (USNC, not seen, cited by Gale). "Baton Rouge": Featherman s.n. (LSU \#29086). Bienville Par.: Reid 6217 (LSU); Reid et al. 6233; 6237 (both LSU). Caldwell Par.: Thomas 4219; 4270 (both VDB). Catahoula Par.: Brown 7383 (LSU). Claiborne Par.: Allen \& Vincent 8417 (BRIT). DeSoto Par.: Brown 6093 (LSU); Ajilvsgi 6293 (BRIT). Grant Par.: Hyatt 9604 (TEX); Hyatt 7681 (LSU); Douglas et al. 1064 (LSU). Jackson Par.: Carter 2553 (VDB). La Salle Par.: Thomas \& Laird 50090 (VDB). Lincoln Par.: Brown 6048 (LSU); Kral 15966 (VDB). Natchitoches Par.: Correll \& Correll 9988 (LSU); Smith 97 (LSU); MacRoberts \& MacRoberts 481 (VDB); MacRoberts \& MacRoberts 878 (VDB); Chance 462 (VDB); Kral 20687 (VDB); Hyatt 9728 (LSU). Ouachita Par.: Thomas 21398 (VDB); Thomas 40817 (BRIT, LSU, NCU, UARK); Thomas 105,749; 106,347 (both VDB); Kral 15751 (VDB). Rapides Par.: Thomas 40834 (NCU); Bruser 371 (LSU); Brown 6125 (LSU); Slaughter 4014; 4086 (both LSU); Arnett \& Hastings 393 (LSU); Correll \& Correll 9911 (LSU). Red River Par.: Ajilvsgi 4781 (BRIT); Thomas 5769 (VDB). Sabine Par.: Letterman s.n. (cited by Gale). St. Helena Par.: Allen 1729; 1745 (both LSU). Union Par.: Kral 24557 (VDB); Brooks 17624 (VDB). Vernon Par.: Allen \& Allen PLK0496 (OKL); Ferguson \& Karaman-Castro 666 (LSU); Thieret 26922 (BRIT); Thieret 30152 (SMU); Chance 1613; 1662; 1683 (all VDB); Allen \& Chance 19045 (VDB). Webster Par.: Brown 5317 (LSU); Urbatsch 10195 (LSU); Kral 27288 (VDB); Hyatt 9584 (LSU). Unknown Par.: "on Red River," Louisiana, Hale s.n. (LSU). OKLAHOMA. Atoka Co.: Penfound P-140 (OKL); Hoagland \& Buthold AB7601 (OKL); Taylor 27934 (BRIT); Taylor \& Taylor 14628 (BRIT). Bryan Co.: Taylor \& Taylor 8221; 10943 (both BRIT); Taylor 26899 (BRIT). Choctaw Co.: Hoagland \& Buthold AB7601 (OKL); Taylor 17165; 20433; $22877 ; 23618$ (all BRIT). Creek Co.: Hoagland et al. DFX439 (BRIT, OKL). Latimer Co.: Means, Jr. 589 (LL). Le Flore Co.: Stevens 2681 (OKL); Correll E Mitchell 34365 (LL); Kral 24608 (VDB), Taylor \& Taylor 26903 (BRIT). Marshall Co.: Goodman 7234 (SMU); Goodman 6883 (OKL, UARK); Penfound s.n. (OKL, UARK). McCurtain Co.: Buthold \& Hoagland AB9942 (OKL); Waterfall 8441 (OKL, TEX); Waterfall 9859 (SMU); Buthold \& Hoagland LR748 (OKL); Buthold \& Hoagland RS-136 (OKL); Correll \& Correll 35933; -959 (both LL); Taylor \& Taylor 11029 (BRIT); Taylor \& Taylor 25294 (BRIT); Raffaelli s.n. (SMU). Pontotoc Co.: Penfound P-343 (BRIT). Pushmataha Co.: Waterfall 8401 (OKL, TEX); Waterfall 11142 (SMU, TEX); Buthold \& Hoagland AB7882 (OKL); Hoagland \& Buthold AB7595 (OKL); Correll \& Mitchell 34314 (LL); Mitchell 3844 (LL); Taylor 24945, -948 (both BRIT); Taylor \& Taylor 8206 (BRIT); Magrath \& Mather 16282 (OKL). TEXAS. Anderson Co.: MacRoberts \& MacRoberts 3411 (BRIT); MacRoberts $\&$ MacRoberts 3686 (TEX); Lodwick 484; 492 (both BRIT); Mayfield E Mendenhall 580 (VDB). Angelina Co.: Correll 34913 (LL); Mahler 9881 (BRIT); Boon 378 (TEX). Bastrop Co.: Carr 7745 (BRIT, TEX); Carr 9283 (BRIT); Carr 31060 (TEX); Carr 34186 (TEX). Bowie Co.: Correll 33395 (LL). Brazos Co.: Kessler 5161 (VDB); Massey 190 (LL). Cass Co.: Correll \& Mitchell 34462 (LL). Cherokee Co.: Ajilvsgi 4881; 4910 (both BRIT). Freestone Co.: Lundell \& Lundell 13116 (LL); Shinners 15328 (SMU). Gonzales Co.: Tharp s.n. (TEX); Carr et al. 24780 (BRIT, TEX), Webster \& Wilbur 2985 (FSU, SMU). Gregg Co.: York s.n. (TEX); Mitchell 3184; 3171 (both LL). Grimes Co.: Ajilvsgi s.n. (BRIT); Jones \& Jones 1815 (VDB). Hardin Co.: Jones $\&$ Jones 9158 (BRIT); Brown 29870 (BRIT); Watson 3397 (SMU). Harris Co.: Fisher 45 (US, cited by Gale). Harrison Co.: Fleetwood 11932 (BRIT); Cory 57744 (SMU); Kral 90016 (VDB). Henderson Co.: Correll 26696 (LL); Mitchell 4080 (LL); Shinners 19120 (SMU); MacRoberts \& MacRoberts 4012 (BRIT). Houston Co.: Palmer 14436 (MO, US, not seen, cited by Gale). Jasper Co.: Correll 28647 (LL); Correll \& Correll 38209 (LL); Correll \& Correll 38210 (LL); Orzell \& Bridges 11075 (TEX); Shinners 24239 (SMU); Carr 17678 (TEX). Leon Co.: Cory 25256 (cited by Gale); Singhurst \& Bridges 13354 (TEX); Nixon 18173 (BRIT). Liberty Co.: Watson 2322; 2432 (both SMU). Marion Co.: Correll 30170 (LL); Correll 26394 (LL); Kral 90027 (VDB); Ajilvsgi 7897 (BRIT). Milam Co.: Rosen \& Jones 411 (LSU). Nacogdoches Co.: Orzell \& Bridges 11101 (TEX). Newton Co.: Orzell \& Bridges 11026 (TEX); Allen et al. 20264; 20304; 20306; 20398; 21144; 21157; 22011 (all LSU); Allen et al. 21086 (BRIT, LSU); Allen et al 21130 (BRIT, LSU); Allen et al. 22156 (LSU, TEX); Allen \& Pastorek 22041 (LSU, TEX); Jones \& Jones 9170 (BRIT). Orange Co.: Letterman s.n. (MO, not seen, cited by Gale). Panola Co.: Orzell \& Bridges 8414 (BRIT, TEX). Polk Co.: Palmer 6781 (MO, not seen, cited by Gale); Orzell \& Bridges 8445 (TEX); Watson 2700 (SMU). Robertson Co.: Brady 13736 (TEX); Starbuck 1070 (BRIT). Ruskin Co.: Shinners 19059 (SMU). San Augustine Co.: Orzell \& Bridges 8391 (NCU, TEX); Cory 56541 (SMU); Shinners 30289 (SMU). San Jacinto Co.: Turner 146 (TEX). Smith Co.: Reverchon s.n. (MO, cited by Gale); Shinners 15727 (SMU); Carr 29818 (TEX). Tyler Co.: Carr 23648 (TEX); Tharp et al. 51-1468 (SMU, TEX); Correll \& Correll 33490 (LL); Watson 2264; 2857 (both SMU). Upshur Co.: Cory 25659 (not seen, USNC, cited by Gale). Van Zandt Co.: Orzell \& Bridges 11381 (TEX). Walker Co.: Young s.n. (MO, not seen, cited by Gale). Wood Co.: Reverchon s.n. (MO, cited by Gale); Mears 904 (TEX); Cory 57656 (SMU); Orzell \& Bridges 8056 B (TEX).

Specimens examined of uncertain or equivocal identity (with comments):

ARKANSAS. Searcy Co.: Rumley Road at Van Buren County line, 9 Sep 1993, Beer 93-145 (UARK). [The achene body width is 1.0-1.1 mm and the beak is 1.5-1.7 mm]. LOUISIANA. Evangeline Par.: Quercus-Nyssa-Pinus bottomlands 4 mi E of Barber, 6 Oct 1960, Ewan 19981 (LSU, VDB). [The achene body width is $1.0 \mathrm{~mm}$ and the beak is $1.9 \mathrm{~mm}$ ]. Livingston Par.: Allen $\&$ Vincent 9252 (NCU). [The achene body width is $1.2 \mathrm{~mm}$ and the beak is $1.4 \mathrm{~mm}$ ]. TEXAS. Gregg Co.: near Kilgore, $30 \mathrm{Jul}$ 1943, Barkley 13508 (TEX). [The achene body is $1.2 \mathrm{~mm}$ wide and the beak is $1.6 \mathrm{~mm}$ long]. Tyler Co.: wet sandy loam in faux bog on alluvial flat, SE corner of Timber Lakes Tract, 8 Oct 2004, Carr 23647 (TEX). [The achene body is $0.9-1.0 \mathrm{~mm}$ wide and the beak is $1.8-2.0 \mathrm{~mm}$ long]. 


\section{DRYOPTERIDACEAE}

DRYOPTERIS: First report of D. celsa for Mississippi, filling in a distribution gap

Primary authors: John C. Kees and Alan S. Weakley

Dryopteris celsa (W. Palmer) Knowlton, W. Palmer, \& Pollard (Dryopteridaceae) is a semi-evergreen fern native to the eastern United States from New York and Michigan south to Georgia, Alabama, Louisiana, and ne. Texas (Montgomery \& Wagner 1993; Kartesz 2015). It is nowhere common, patchily distributed across this broad range, and considered to be of conservation concern ( $\mathrm{S} 1, \mathrm{~S} 2, \mathrm{~S} 3$, or $\mathrm{SH}$ ) in the great majority of the twenty-one states in which it occurs (NatureServe 2018). It has been demonstrated that D. celsa is a fertile allotetraploid species, derived by polyploidization from original hybridization between $D$. goldieana (Hook. ex Goldie) A. Gray, currently distributed widely in northeastern North America, and D. ludoviciana (Kunze) Small, currently distributed in the southern Coastal Plain from northeastern North Carolina south to southern peninsular Florida, and west to eastern Texas. The two parent species currently have essentially allopatric distributions, and in some areas D. celsa co-occurs with one parent, while in other areas the species grows where neither parent presently occurs (e.g., the Coastal Plain from New Jersey to Virginia) (Montgomery \& Wagner 1993; BONAP 2015).

We here report what we believe to be the first documented occurrence of D. celsa in Mississippi.

U.S.A. MISSISSIPPI. Lincoln Co.: Brookhaven, private property, 329 Big Springs Dr. NE, Brookhaven, MS 39601, hydric, swampy forest near the Little Fair River (and adjacent Pinus taeda pine plantation) dominated by Nyssa aquatica and Magnolia virginiana, also with Osmundastrum cinnamomeum and Polystichum acrostichoides, 31.5685 N -90.2950 E, 99 m elev., 12 May 2018, John C. Kees 1 (NCU 658274, NCU 658275, NCU 658276).

This report of Dryopteris celsa is the first known documentation for Mississippi, expanding the range of the species to 22 states in the eastern United States. The plants were first observed in the winter of 2017 from persistent sterile fronds, and the fern was identified as D. celsa on April 8, 2018. Specimens were taken May 12 from the same site and sent to the UNC-CH Herbarium at the University of North Carolina at Chapel Hill. The nearest reported populations are in Morehouse Parish, Louisiana, approximately 127 miles or 204 kilometers distant (to the northwest and across the Missisippi River in the West Gulf Coastal Plain) and in Monroe County, Alabama, approximately 166 miles or 267 kilometers distant (to the east, and in the East Gulf Coastal Plain).

The population of Dryopteris celsa was found in a habitat that is a mix of hydric, swampy forest, dominated by Nyssa aquatica L. and Magnolia virginiana L., and pine plantation (Fig. 6). The area could have been cypress swamp previously, as suggested by a few remnant Taxodium distichum (L.) Rich. saplings, but the area was logged and the main stream was channelized for cattle fields; later, around 40 years ago, the area was planted with Pinus taeda $\mathrm{L}$. The population of $D$. celsa in the area is now most concentrated along the channelized stream, associated with the edges of the pine plantation, whereas the hardwood portion of the area, which has a higher water table, is instead primarily occupied by Osmundastrum cinnamomeum (L.) C. Presl.

\section{ONAGRACEAE}

Oenothera: Reassessment of Oenothera fruticosa var. unguiculata Primary authors: Bruce A. Sorrie, Richard J. LeBlond, and Alan S. Weakley

Fernald (1939) described a near-coastal variant within the widespread Oenothera fruticosa L. as O. fruticosa var. unguiculata Fernald. He stated that "Var. unguiculata in its long sepal-tips is quite unlike the other varieties of Oenothera fruticosa." In his amended description (Fernald 1950), he stated “... calyx appressed-strigose, the tips free, arching, subulate, and claw-like, 1-2.5 mm long; capsule slenderly obconic, very sulcate (grooved), spreading-villous, the body $4-10 \mathrm{~mm}$ long, stipe to $1.5 \mathrm{~cm}$ long." These values contrast with those given for var. fruticosa: calyx tips "at most about $1 \mathrm{~mm}$ long" and not divergent or arched.

Munz (1965) accepted variety unguiculata but provided no new insights. Straley (1977) did not recognize variety unguiculata and instead subsumed it within his subspecies fruticosa. He stated that "... populations 


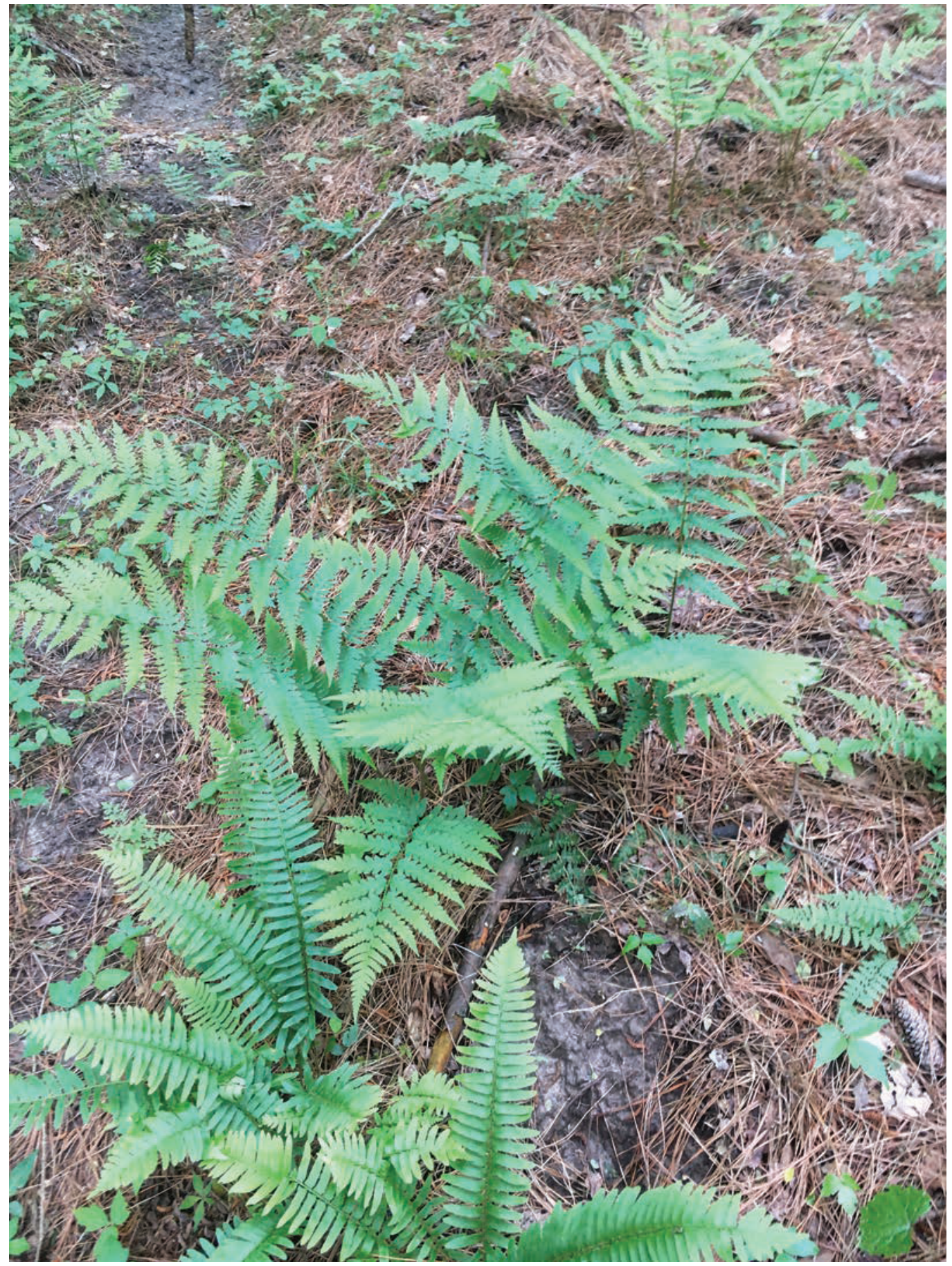

FIG. 6. Dryopteris celsa in Lincoln County, Mississippi, Other ferns present and visible include Polystichum acrostichoides and Athyrium asplenioides. 8 April 2018. Photo by J.C. Kees. 
along the coast of southern North Carolina and adjacent South Carolina frequently have sepal tips elongate, up to $6 \mathrm{~mm}$ and quite divergent."

We assessed the characters used by Fernald $(1939,1950)$ and measured 20 examples of sepal tips and capsule length of specimens at NCU. Capsule body length ranged from 6-11 mm, as compared to Fernald's range of 4-10 mm. Sepal tips ranged from (1-)1.5-2.5(-3) mm, a bit longer than Fernald's range of 1-2.5 mm. Sepal tips in bud are best described as arched-ascending or incurved-ascending. After the sepals have reflexed, the tips are better described as often arching away from the plane of the sepal. The dark amber color and cartilaginous texture of the tips is visible and can be assessed when the sepals are reflexed.

Calyx pubescence differs among the taxa within $O$. fruticosa. In var. unguiculata, pubescence is appressedstrigose, whereas in other taxa it varies from coarsely pilose to fine and strigillose.

We had hoped to compare petal length as well, for it is our distinct impression in the field that corollas are larger in var. unguiculata than in nominate var. fruticosa, but the majority of herbarium specimens we examined at NCU have shriveled or misshapen corollas. Five petals measured 19, 20, 22, 22, and $25 \mathrm{~mm}$, vs. 16-19 mm in var. fruticosa, which lends credence to our field observations.

Finally, we separated out those specimens identified as Oenothera riparia Nutt., as per Weakley (2015) and Wagner et al. (2007). While included in var. fruticosa by Fernald, its elongate sepal tips are like those of var. unguiculata and may cause confusion during identification. Wagner et al. (2007) stated: "We agree with the conservative approach used by Straley (1977) in recognizing a broadly delimited O. fruticosa, except that we recognize the very distinctive semi-aquatic octoploid populations (Straley 1982) as O. riparia. The plants of these coastal populations, which occur from southern Virginia to North Carolina, are more robust, more branched, and less pubescent than those of the two subspecies of $O$. fruticos $a$, and have slightly succulent leaves and more prominent adventitious roots (Straley 1982; D. Boufford, pers. comm.)." Oenothera riparia is much taller than $O$. fruticosa var. unguiculata (7-12 dm) and has much longer capsules (15-20 mm). It occurs in blackwater rivers from southeastern Virginia to southeastern South Carolina on floating logs, emergent stumps, and at margins of seasonally inundated swamp forests.

The markedly long and often curved sepal tips, appressed-pilose sepals, larger petals, and strictly Coastal Plain distribution (maritime or near-maritime, and in calcareously influenced wet savannas) argue for species status for var. unguiculata. This requires a new combination:

Oenothera unguiculata (Fernald) Sorrie, LeBlond, \& Weakley, comb. et stat. nov. (Fig. 7). BAsIonym: Oenothera fruticosa L. var. unguiculata Fernald, Rhodora 41(491):551, pl. 577, f. 1-3. 1939. TYPE: U.S.A. VIRGINIA. Greensville Co.: dry pine woods south of Skipper's, velvety, sepal tips prolonged, 21 May 1939, M.L. Fernald \& B. Long 9991 (HоLоTYPE: GH!, ISOTYPE: PH).

Oenothera unguiculata belongs to an interesting group of species that occurs in calcareously influenced wet savannas and maritime wet grasslands on the outer Coastal Plain, including Rhynchospora pinetorum Britton \& Small, Scleria verticillata Muhl. ex Willd., and Dichanthelium caerulescens (Hack. ex Hitchc.) Correll (Fig. 8).

The recent recognition of $O$. unguiculata and $O$. riparia provides incremental progress towards clarifying the Oenothera fruticosa complex. Recognition of var. microcarpa Fernald of boggy depressions in the Coastal Plain, var. subglobosa (Small) Munz of granitic flatrocks, subsp. glauca (Michx.) Straley of the Southern Appalachians, and other potential segregates remains uncertain, awaiting additional study so that their proper disposition can be determined.

Specimens examined: U.S.A. GEORGIA. Cook Co.: Faircloth 5835 (NCU) [Sepal tips $4.0 \mathrm{~mm}$ long. While disjunct and inland, this specimen matches in all respects those from the Carolinas and Virginia]. NORTH CAROLINA. Beaufort Co.: pine forest 4 mi N of Wilmar, 17 May 1958, Radford 33378 (NCU). Carteret Co.: Atlantic, powerline on Duke property, 5 Jun 1992, LeBlond sight record. Dare Co.: Pea Island National Wildlife Refuge, W of NC 12 and N of Rodanthe, wet maritime grassland, 17 May 2012, Sorrie 12961 (NCU). Hyde Co.: ditch 4.2 mi SW of New Holland, 6 Aug 1958, Radford 38991 (NCU). Jones Co.: S of Coombs Fork, 28 Jul 2001, LeBlond 5529 (NCU). New Hanover Co.: sands behind dune area SW of Fort Fisher, 12 Jun 1958, Bell 12947 (NCU); Carolina Beach SP, open boggy waste, full sun, with Baccharis halimifolia, 21 May 1949, Browne, Jr. S-357 (NCU); bank of drainage ditch, Carolina Beach, 18 Apr 1938, Godfrey \& Buell 3535 (NCU). Onslow Co.: roadside 7.2 mi W of jct NC 53 and US 258, on 53, 28 Apr 1957, Ahles \& Ramseur 24141 (NCU); Haws Run Mitigation Site natural area, 25 Jun 1996, LeBlond 4575 (NCU). Pender Co.: Shaken Creek Savanna natural area, 25 May 1998, LeBlond 4976 (NCU); The Neck Savanna, TNC tract, 26 May 1998, LeBlond 4978 (NCU); McLean Savanna, 20 Jun 2007, LeBlond 6419 (NCU); Moore's Creek National Battlefield, near base of slope behind visitor center, 26 May 2003, LeBlond 5758 (NCU). SOUTH CAROLINA. Berkeley Co.: pine savanna 


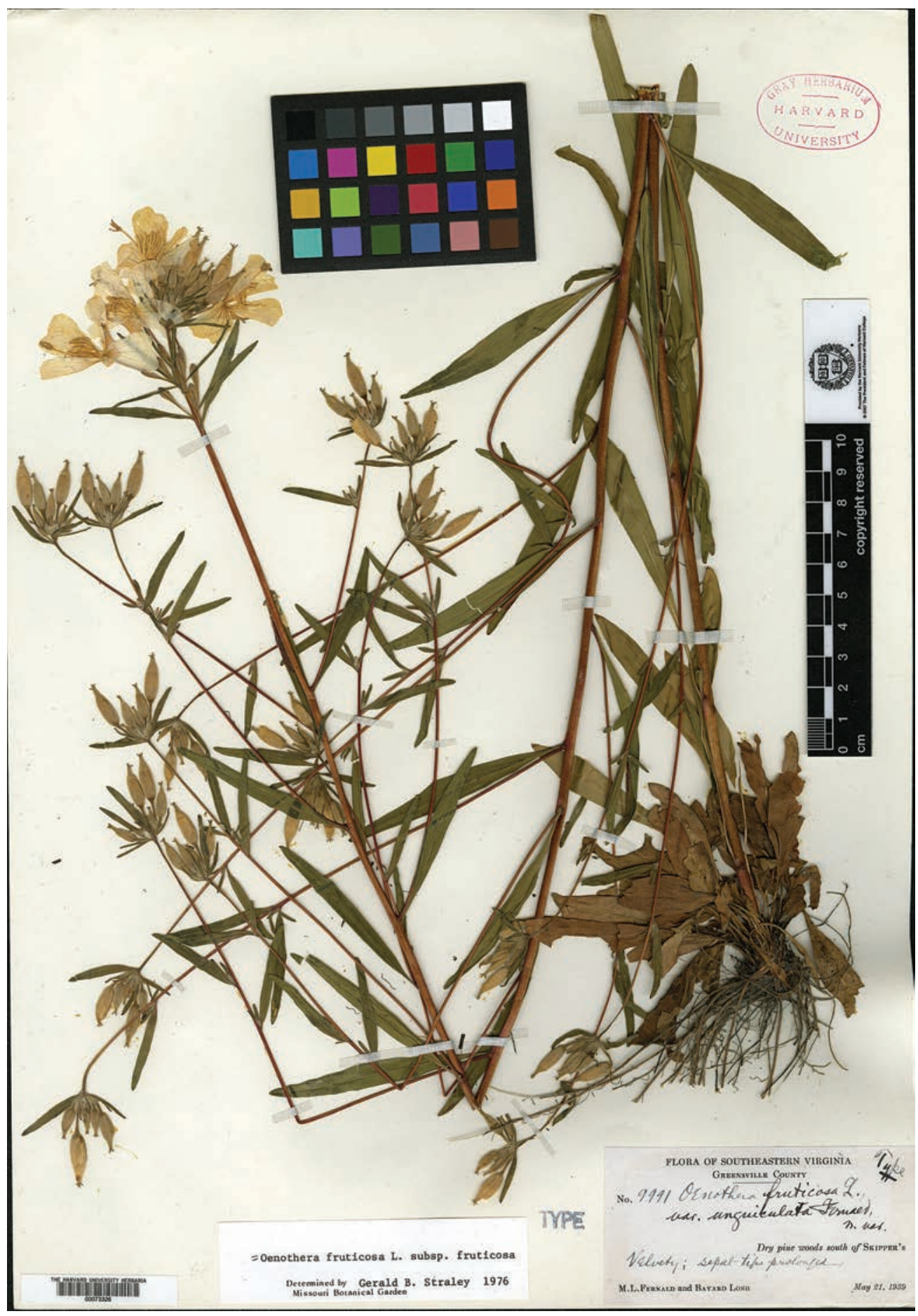

FIG. 7. Holotype of Oenothera fruticosa var. unguiculata (GH). 


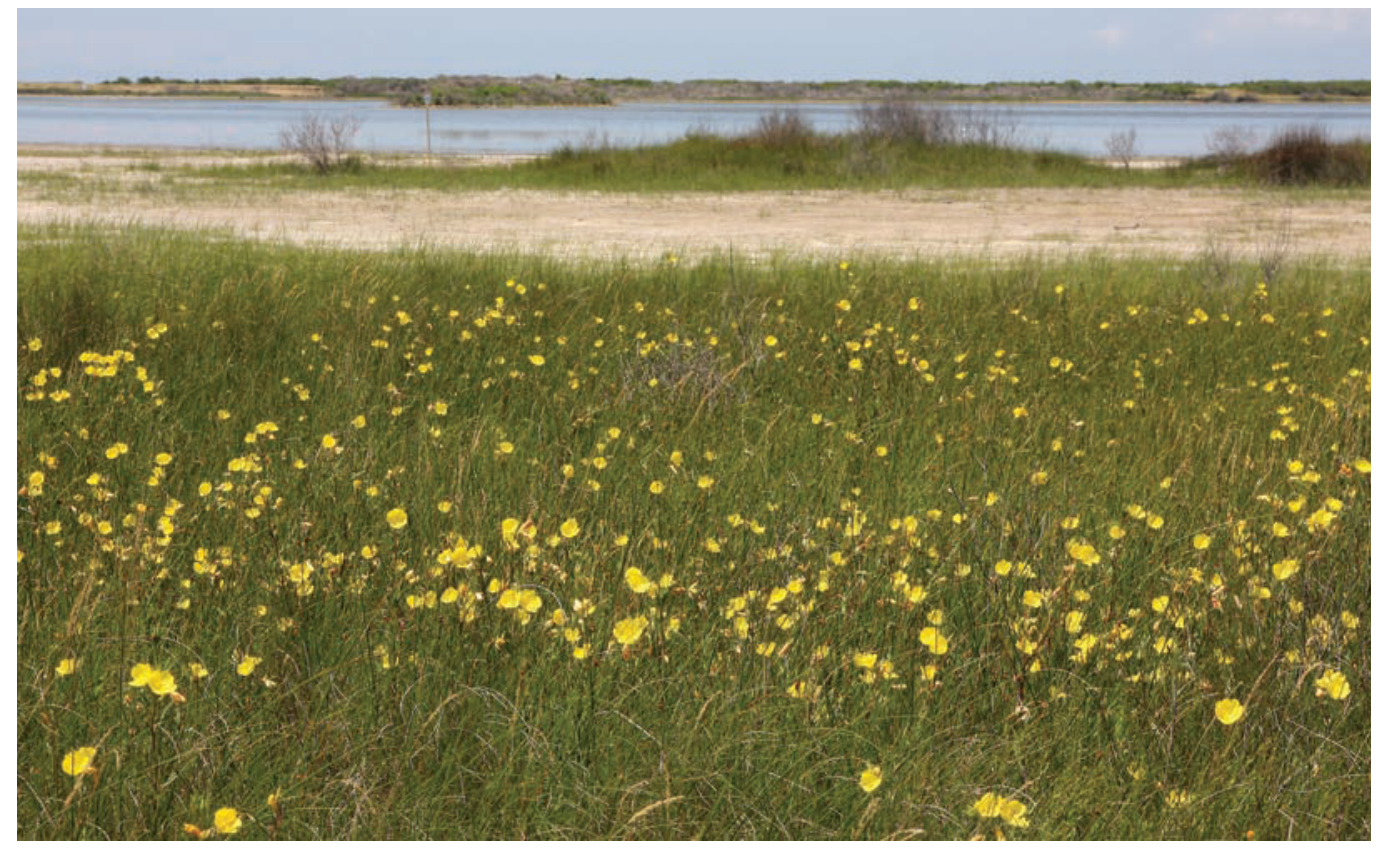

FIG. 8. Oenothera unguiculata in wet maritime grassland, Dare County, NC. 2 June 2013, B.A. Sorrie.

just N of Russellville on CR 35, 7 Apr 1957, Ahles \& Haesloop 22422 (NCU). Clarendon Co.: ditch 2.5 mi SSW of Summerton, 20 Apr 1957, Radford 21238 (NCU). Georgetown Co.: roadside 2.5 mi NW of Campfield, 22 Apr 1957, Radford 21618 (NCU). Hampton Co.: open area along railroad, $3.1 \mathrm{mi}$ NW of Yemassee on SC 28, 11 May 1956, Ahles $\&$ Bell 12430 (NCU). Marion Co.: open sandy roadside and woodland margins at jct between Britton Neck and Centenary, 21 Apr 1957, Bell 6371 (NCU). Richland Co.: low grounds, 16 Jun 1855, Hexamer \& Maier s.n. (GH, cited by Fernald). VIRGINIA. Greensville Co.: dry pine woods S of Skipper's, 21 May 1939, Fernald E Long 9991 (holotype GH!, isotype PH); same place 18 Jul 1939, Fernald \& Long 10747 (GH). Sussex Co.: peaty and argillaceous swale N of Littleton, 22 May 1939 , Fernald \& Long 9990 (GH).

\section{POACEAE}

Eragrostis: Typification of Eragrostis refracta and E. spectabilis Primary author: Alan R. Franck

Eragrostis Wolf (Poaceae) is a globally distributed genus comprising ca. 350 species. One group of four similar species native to the southeastern USA includes E. elliottii S. Watson, E. hirsuta (Michx.) Nees, E. refracta (Muhl.) Scribn., and E. spectabilis (Pursh) Steud. This group is characterized by extremely diffuse inflorescences to $45 \mathrm{~cm}$ wide and $60 \mathrm{~cm}$ long, with the spikelets very distant from each other and separated by long inflorescence branches, internodes, and pedicels (the pedicels or internodes up to $4.5 \mathrm{~cm}$ long). Misidentifications are relatively frequent, but presence or absence of rhizomes, pubescence of leaf sheaths and inflorescence nodes, pedicel orientation, and number of florets per spikelet are useful for identification (Table 2). Two names, E. refracta and E. spectabilis, have somewhat brief validating descriptions for their basionyms and require typification to secure their application.

Eragrostis refracta is endemic to the southern and eastern USA (Hitchcock 1935: 851-852; Harvey 1948; Hitchcock 1951: 869; Peterson 2003). An early description of the species was probably provided by Walter (1788), who misapplied the name Poa amabilis L. (J. McNeill, pers. comm.). Walter's description included "panicula diffusa" and "remotis quindecimfloris" which fit the current concept of E. refracta (Peterson 2003).

The basionym P. refracta Muhl. was validly published by reference (Turland et al. 2018: Art. 38) to "Poa amabilis Walt.," although nearly all other new names introduced in Muhlenberg (1813) are invalid. Elliott's 
TABLE 2. Overview of four species of Eragrostis with extremely diffuse inflorescences that are native to the southeastern U.S.A.

\begin{tabular}{|c|c|c|c|c|c|}
\hline & Habit & Upper culm leaf sheaths & Lower inflorescence nodes & Pedicel orientation & Florets per mature spikelet \\
\hline E. elliottii & cespitose & glabrate & sparsely pilose & $\begin{array}{l}\text { ascending to spreading, } \\
\text { occasionally some appressed }\end{array}$ & $9-15(30)$ \\
\hline E. hirsuta & cespitose & densely pilose & glabrate & $\begin{array}{l}\text { ascending to spreading, } \\
\text { occasionally some appressed }\end{array}$ & $2-4(6)$ \\
\hline E. refracta & cespitose & glabrate & $\begin{array}{l}\text { sparsely to moderately } \\
\text { pilose }\end{array}$ & $\begin{array}{l}\text { appressed, almost none } \\
\text { ascending or spreading }\end{array}$ & $4-22(30)$ \\
\hline E. spectabilis & $\begin{array}{l}\text { branched } \\
\text { rhizomes }\end{array}$ & densely pilose & $\begin{array}{l}\text { moderately to densely } \\
\text { piloset }\end{array}$ & $\begin{array}{l}\text { ascending to spreading, } \\
\text { occasionally some appressed }\end{array}$ & $3-10(12)$ \\
\hline
\end{tabular}

(1816) later description of $P$. refracta is not the first valid publication of the name as is sometimes thought (Harvey 1948; Peterson et al. 2001: 107). Although Lamson-Scribner (1894: 49) incorrectly cited the basionym as being from Muhlenberg (1817: 146), the combination E. refracta can be considered validly published by Scribner (Turland et al. 2018: Art. 41.3).

Since the validating description for E. refracta is the description of the misapplied Poa amabilis sensu Walter, typification of E. refracta should be based on any original material used by Walter (Turland et al. 2018: Art. 7.8). No extant original material is known for this species (Hitchcock 1905; Ward 2007a, 2017). To attempt to typify E. refracta as accurately as possible and maintain consistency with its modern usage, a neotype is here designated that was collected relatively near Walter's plantation, although occasionally Walter's descriptions must have been based on material from far outside his plantation (Ward 2007b).

Eragrostis refracta (Muhl.) Scribn., Mem. Torrey Bot. Club 5:49. 1894. Poa refracta Muhl., Cat. Pl. Amer. Sept. 12.1813. TYPE: U.S.A. SOUTH CAROLINA. Charleston Co.: Santee Coastal Reserve, Washo Reserve, W end along C-71, S of swampy creek crossing, South Santee, 11 Sep 1992, S.R. Hill 24298 (NEOTYPE, designated here: NY barcode 1700232; ISONEOTYPES: MO barcode 850299, USF acc. no. 206975).

Eragrostis spectabilis is native to southern and eastern USA, Mexico, and Belize (Hitchcock 1935: 852-853; Harvey 1948; Hitchcock 1951: 870; Peterson 2003; Peterson \& Valdés-Reyna 2005). Its basionym, Poa spectabilis Pursh, is legitimate even though Pursh (1813) cited Walter's misapplication of Poa amabilis L.; Poa amabilis sensu Walter was untypified at the time and was not published as a later homonym, so Art. 52.2 (Turland et al. 2018) does not apply. Walter's misapplied P. amabilis provides the validating description for E. refracta, discussed above, and therefore must also provide the type of that name, but Pursh (1813) provided a separate description for P. spectabilis. He described the spikelets as "decemfloris," consistent with the current concept of E. spectabilis (Peterson 2003).

The type of E. spectabilis has been considered to be Clayton 580 (Peterson \& Valdés-Reyna 2005) although it appears this specimen has not yet been validly designated as the type (Turland et al. 2018: Art. 7.11). This Clayton 580 specimen was indirectly cited in the protologue of a different species, P. capillaris L. (= Eragrostis capillaris (L.) Nees), through reference to Gronovius, who first cited Clayton 581 (1743: 36) and later cited Clayton 580 in the second edition of his work (Gronovius 1762: 13). The Latin text on the Clayton 580 (BM) specimen matches that found in Gronovius $(1743,1762)$ and the protologue of P. capillaris. However, P. capillaris was later lectotypified by a different specimen and is not conspecific with E. spectabilis (Hitchcock 1908: 121).

The specimen Clayton 580 is identifiable with the current concept of E. spectabilis (Peterson 2003) and the fragment at US was annotated as such by L.H. Harvey and P.M. Peterson. In the protologue of P. spectabilis, Pursh used the abbreviation "v.v." referring to observing the plant in its living state (Pursh 1814: xxi). Since Pursh (1814: xvi-xviii) indicated use of the Clayton Herbarium, Clayton 580 may be considered likely original material available for lectotypification.

Eragrostis spectabilis (Pursh) Steud, Nomencl. Bot., ed. 2, 1:564. 1840. Poa spectabilis Pursh, Fl. Amer. Sept. 1:81. 1813. TYPE: Clayton 580 (LECTOTYPE: designated here: BM barcode 000051631; ISOLECTOTYPE: US barcode 00513255). 


\section{TRILLIACEAE (OR MELANTHIACEAE S.L.)}

Trillidium: Trillium undulatum as the second species of Trillidium

Primary authors: Aaron J. Floden and Edward E. Schilling

Results of phylogenetic analyses of tribe Paridae have consistently resulted in the placement of Trillium undulatum Willd. (Trilliaceae), essentially endemic to the Appalachian Mountains of eastern North America, as sister to the monotypic Trillidium govanianum Wall. ex Royle, endemic to the northwestern Himalaya (Chupov et al. 2007; Farmer 2006; Farmer \& Schilling 2002; Kim et al. 2016; Osaloo et al. 1999; Osaloo \& Kawano 1999; Pellicer et al. 2014). Its placement outside Trillium has been attributed to long branch attraction (Farmer 2006), but additional studies based on larger sampling of both taxa and sequence data-including whole plastid sequence data-have consistently recovered the sister group relationship of Trillium undulatum and Trillidium govanianum (Schilling et al., in prep.).

Gates (1917) considered the morphology of Trillium undulatum to be peculiar amongst the other Trillium: the leaves are distinctly petiolate, the petals are colored with purple-red in characteristic stripes on the proximal portions of the petals (except in odd formas), and the margins of the petals are undulate (this latter character not unique in Trilliaceae) (Fig. 9). In addition, the anthers are subequal to the filaments and shorter than the stigmas, which are connate and form a short distinctive column before the three stigmatic branches diverge. Most distinctive in comparison to other Trillium species, T. undulatum has bright red to scarlet, unridged, berry-like, cylindrical fruits that differ from any found within Trillium (Zomlefer 1996; Fig. 9). Other species of Trillium that have pedicellate flowers have typically strongly ridged fruit that can be reddish-purple in color, are ovoid, globose or pyramidal, but the fruit is not as distinctly baccate as T. undulatum (Case \& Case 1997; Zomlefer 1996).

Comparison of the heterochromatin banding in the karyotypes of Trillium and related genera shows that T. undulatum is most similar to Trillidium govanianum. Fukuda (2001b) reported $2 n=2 x=10$ for T. undulatum; he also found that the heterochromatin banding of T. undulatum is more similar to Trillidium govanianum, which is an allotetraploid $(2 n=4 x=20)$, than to other Trillium species (Haga \& Watanabe 1966).

Pollen studies of the Trilliaceae also reveal similar surface morphology between Trillidium govanianum and Trillium undulatum, although the former is monocolpate and the latter inaperturate (Takahashi 1982, 1983). Pollen of Trillidium govanianum is monocolpate and intectate (Takahashi 1983) and differs from species of Paris s.l. (incl. Daiswa and Kinugasa), which are ellipsoidal and monosulcate (Takahashi 1984) and most Trillium, which are inaperturate (Takahashi 1982, 1983). Pollen of T. undulatum is of the verrucate type composed of a verrucate exine ornamentation and unique within Trillium including those from eastern Asia (Takahashi 1982, 1983). The exine ornamentation of T. undulatum and Trillidium govanianum is similar, with verrucae that are striately sculptured and angulate (Wei 1995). These two species have pollen ornamentation that is distinct from species of Paris s.l. and also from Trillium, which further supports considering Trillium undulatum and Trillidium govanianum to be congeneric. The three prominent colored petals give Trillium undulatum the superficial appearance of a typical Trillium, but other evidence shows it is part of a phylogenetically distinct lineage, a situation reminiscent of the case of Pseudotrillium rivale (Farmer \& Schilling 2002). Although there are also striking morphological differences between Trillium undulatum and Trillidium govanianum, notably in the petal appearance and features of the stigma, it appears to be preferable to treat the two species as composing a single genus. Here we provide a new combination to transfer Trillium undulatum to Trillidium.

Trillidium undulatum (Willd.) Floden \& Schilling, comb. nov. BAsIOnym: Trillium undulatum Willd. Der Gesellsschaft Naturforschender Freunde zu Berlin, neue Schriften 3:422. 1801. TyPe: NORTH AMERICA. U.S.A.: Habitat in Pennsylvania ("Wächst in Pensylvanien") (LECTOTYPE, designated here: B, B-W 07091-010).

Trillium erythrocarpum Michx., Fl. Bor. Amer. 1:216. 1803.

Trillium pictum Pursh, Fl. Amer. Sept. 244. 1814. 

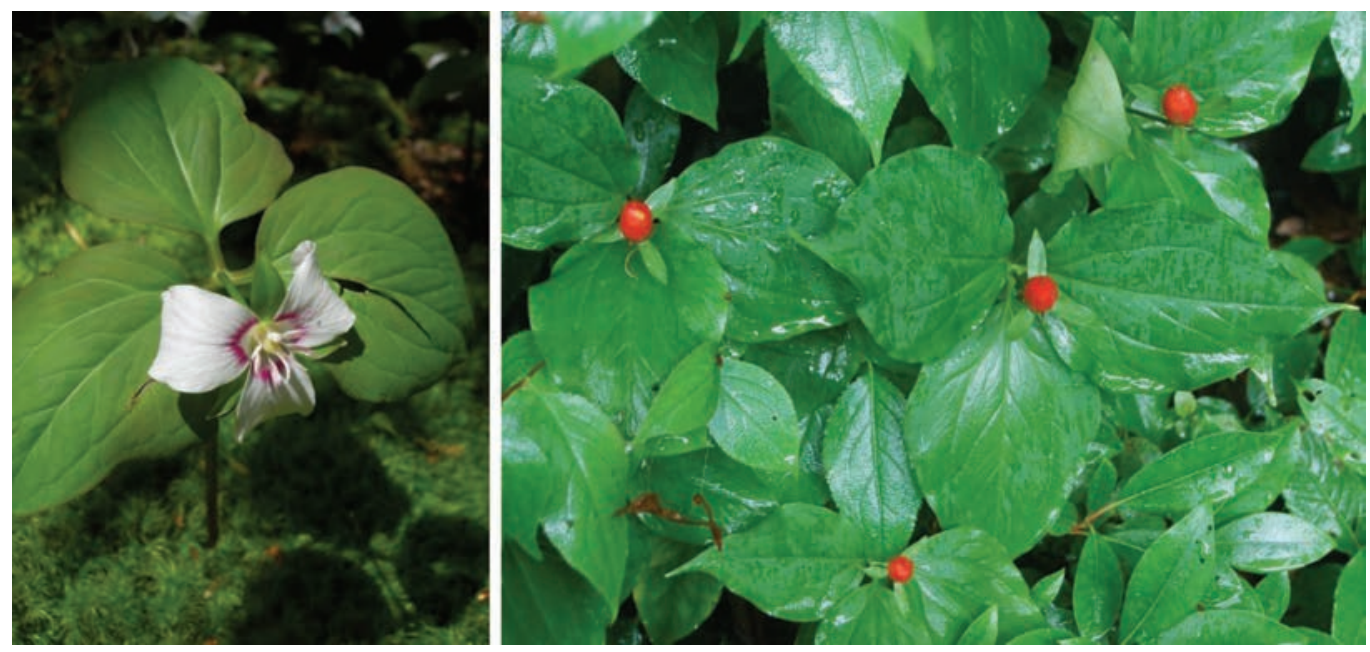

FIG. 9. Trillidium undulatum: left, flowering in Cocke Co., Tennessee, USA showing the distinctive petiolate leaves and flowers with red-marked tepals; right, fruiting in Sevier Co., Tennessee, USA, showing the distinctive red berry-like fruits, photographs by A.J. Floden.

\section{ACKNOWLEDGMENTS}

We thank the curators and collections managers of the following herbaria for loans and physical/digital access to specimens: B, BM, BRIT, DUKE, FSU, FTG, LL, LSU, MICH, MO, NCU, NY, OKL, SMU, TEX, UARK, US, USF, USNC, and VDB@BRIT. The manuscript was improved by reviews by Guy Nesom and Melanie Schori, but the authors take responsibility for any remaining limitations. Derick Poindexter prepared the images of achenes for Rhynchospora. The Edward Swab Fund for Floristic Botany at the University of North Carolina Herbarium provided support for some of this work.

\section{REFERENCES}

BLAKE, S.F. 1918. Notes on the Clayton herbarium. Rhodora 20:21-28.

CASE, F.W. JR. \& R.B. CASE. 1997. Trilliums. Timber Press, Portland, Oregon, U.S.A.

CHupov, V.S., E.O. PUnINA, E.M. MACHS, \& A.V. Rodionov. 2007. Nucleotide composition and CpG and CpNpG Content of ITS1, ITS2, and the 5.8S rRNA in representatives of the phylogenetic branches Melanthiales-Liliales and MelanthialesAsparagales (Angiospermae, Monocotyledones) reflect the specifics of their evolution. Molec. Biol. 41(5):737-755.

CoRRELL, D.S. \& M.C. Johnston. 1970. Manual of the vascular plants of Texas. Texas Research Foundation, University of Texas at Dallas, U.S.A.

Diggs, G.M., JR., B.L. LIPsComb, M.D. Reed, \& R.J. O'Kennon. 2006. Illustrated flora of East Texas. Vol. 1. Botanical Research Institute of Texas, Fort Worth, U.S.A.

ELLIOT, S. 1816. A sketch of the botany of South-Carolina and Georgia. J.R. Schenck, Charleston, South Carolina, U.S.A.

FARMER, S.B. 2006. Phylogenetic analyses and biogeography of Trilliaceae. Aliso 22:579-592 http://scholarship.claremont.edu/aliso/vol22/iss1/45

FARMeR, S.B. \& E.E. SchilLing. 2002. Phylogenetic analyses of Trilliaceae based on morphological and molecular data. Syst. Bot. 27:674-692.

FERNALD, M.L. 1939. Last survivors in the flora of Tidewater Virginia. Rhodora 41:465-504, 529-559, 564-574 (pagination interrupted).

FerNALD, M.L. 1950. Gray's manual of botany. Eighth ed. American Book Co., New York, U.S.A.

FukUDA, I. 2001. The origin and evolution in Trillium 1. The origin of the Himalayan Trillium govanianum. Cytologia 66:105-111.

FuKUDA, I. 2001. The origin and evolution in Trillium 2. Chromosome variation of Trillium undulatum in North America. Cytologia 66:319-327. 
Gale, S. 1944. Rhynchospora, Section Eurhynchospora, in Canada, the United States, and the West Indies. Rhodora 46:89-134, 159-197, 207-249, 255-278.

Gates, R.R. 1917. A systematic study of the North American genus Trillium, its variability, and its relation to Paris and Medeola. Ann. Missouri Bot. Gard. 4:43-92.

GENTRY, J.L. ET AL. 2013. Atlas of the vascular plants of Arkansas. University of Arkansas Herbarium, Fayetteville, U.S.A.

Godfrey, R.K. \& J.W. Wooten. 1979. Aquatic and wetland plants of southeastern United States, monocotyledons. University of Georgia Press, Athens, Georgia, U.S.A.

Gronovius, J.F. 1743. Flora Virginica. Cornelium Haak, Leiden, Netherlands.

Gronovius, J.F. 1762. Flora Virginica. Leiden, Netherlands.

Haga, T. \& H. Watanabe. 1966. Chromosomes of a Himalayan Trillium in relation to the Japanese and American species. Proc. Japan Acad. 42:160-164.

HARVEY, L.H. 1948. Eragrostis in North and Middle America. Ph.D. dissertation, University of Michigan.

HІтснсоск, A.S. 1905. The identification of Walter's grasses. Rep. (Annual) Missouri Bot. Gard. 16:31-56.

HITCHсOCK, A.S. 1908. Types of American grasses. Contr. U.S. Natl. Herb. 12:113-158.

HiтсHсOCK, A.S. 1935. Manual of the grasses of the United States. Misc. Publ. U.S.D.A. 200:1-1040.

HiтсHсOCK, A.S. 1951. Manual of the grasses of the United States. Misc. Publ. U.S.D.A. 200:1-1051.

HuANG Y., X. LI, Z. YANG, C. YANG, J. YANG, \& Y. J. 2016. Analysis of complete chloroplast genome sequences improves phylogenetic resolution in Paris (Melanthiaceae). Front. PI. Sci. https://doi.org/10.3389/fpls.2016.01797

JI, Y., P.W. FrITSCH, H. LI, T. XIAO, Z. ZHOU. 2006. Phylogeny and classification of Paris (Melanthiaceae) inferred from DNA sequence data. Ann. Bot. 98:245-256.

KarTeSZ, J.T., The biota of North America Program (BONAP). 2015. North American plant atlas. (http://bonap.net/napa). Chapel Hill, N.C. [maps generated from Kartesz, J.T. 2015. Floristic Synthesis of North America, Version 1.0. Biota of North America Program (BONAP).

KIM, S.-C., J.S. KIM, M.W. CHASE, M.F. FAY, \& J.-H. KIM. 2016. Molecular phylogenetic relationships of Melanthiaceae (Liliales) based on plastid DNA sequences. Bot. J. Linn. Soc. 181:567-584. https://doi.org/10.1111/boj.12405

KRAL, R. 2002. Rhynchospora. Flora of North America north of Mexico. Vol. 23, Magnoliophyta: Commelinidae (in part): Cyperaceae. Oxford University Press, New York, U.S.A.

LAMSON-SCRIBNER, F. 1894. Graminae. Mem. Torrey Bot. Club 5:27-59.

LEBLond, R. 2015. Rhynchospora. In: Weakley, A.S. Flora of the southern and mid-Atlantic states, May 2015. University of North Carolina Herbarium, Chapel Hill, U.S.A..

McMilLan, P.D. 2007. Rhynchospora (Cyperaceae) of South Carolina and the eastern United States. Clemson University Public Service Publishing, Clemson, U.S.A.

Montgomery, J.D. \& W.H. WAGneR, JR. 1993. Dryopteris. In: Flora of North America Editorial Committee. Flora of North America north of Mexico. Volume 2: Pteridophytes and Gymnosperms. Oxford Univ. Press, New York, U.S.A.

Munlenberg, H. 1813. Catalogus Plantarum Americae Septentrionalis. Lancaster, Pennsylvania, U.SA.

MuhlenBERG, H. 1817. Descriptio uberior graminum et plantarum calamariarum Americae Septentrionalis Indigenarum et Cicurum. Solomon W. Conrad, Philadelphia, U.S.A.

Munz, P.A. 1965. Onagraceae. North American flora, ser. 2, 5:1-278.

NACZI, R.F.C. \& R.D. MOYER. 2017. Revision of the Rhynchospora glomerata species complex, focusing on the taxonomic status of $R$. leptocarpa (Cyperaceae). Brittonia 69:114-126.

NatureServe. 2018. NatureServe Explorer: An online encyclopedia of life [web application]. Version 7.1. NatureServe, Arlington, Virginia. Available http://explorer.natureserve.org. (Accessed: September 28, 2018).

Osaloo, S.K., F.H. UteCh, M. Ohara, \& S. Kawano. 1999. Molecular systematics of Trilliaceae I. Phylogenetic analyses of Trillium using matK gene sequences. J. PI. Res. 112:35-49.

Osaloo, S.K. \& S. Kawano. 1999. Molecular systematics of Trilliaceae II. Phylogenetic analyses of Trillium and its allies using sequences of rbcL and matK genes of $\mathrm{cpDNA}$ and internal transcribed spacers of $18 \mathrm{~s}-28 \mathrm{~s}$ nrDNA. PI. Spec. Biol. 14:75-94.

Pellicer, J., L.J. Kelly, I.J. Leitch, W.B. Zomlefer, \& M.F. Fay. 2014. A universe of dwarfs and giants: Genome size and chromosome evolution in the monocot family Melanthiaceae. New Phytol. 201:1484-1497. doi: 10.1111/nph.12617

Peterson, P.M. 2003. Eragrostis. In: Flora of North America Editorial Committee, eds. Flora of North America north of Mexico. 25:65-105. Oxford University Press, New York, U.S.A.

Peterson, P.M. \& J. Valdés-Reyna. 2005. Eragrostis (Poaceae: Chloridoideae: Eragrostideae: Eragrostidinae) from northeastern Mexico. Sida 21:1363-1418. 
Peterson, P. M., R.J. Soreng, G. Davidse, T.S. Filgueiras, F.O. Zuloaga, \& E.J. Judziewicz. 2001. Catalogue of New World grasses (Poaceae): II. subfamily Chloridoideae. Contr. U.S. Natl. Herb. 41:1-255.

PURSH, F. 1813. Flora Americae Septentrionalis, vol. 1. London, U.K.

$\mathrm{S}_{\mathrm{MITH}}$, E.B. 1988. An atlas and annotated list of the vascular plants of Arkansas. Second Edition. Published by the author, Fayetteville, Arkansas, U.S.A.

SoRRIE, B.A. \& A.S. WEAKLEY. 2001. Coastal plain vascular plant endemics: Phytogeographic patterns. Castanea 66:50-82.

Straley, G.B. 1977. Systematics of Oenothera sect. Kneiffia (Onagraceae). Ann. Missouri Bot. Gard. 64:381-424.

StRALEY, G.B. 1982. Octoploid populations of Oenothera fruticosa L. (Onagraceae) from coastal North Carolina. Rhodora 84:281-283.

TAKAHASH, M. 1982. Pollen morphology in North American species of Trillium. Amer. J. Bot. 69:1185-1195.

TAKahashi, M. 1983. Pollen morphology in Asiatic species of Trillium. Bot. Mag. Tokyo 96:377-384. https://doi. org/10.1007/BF02488182

TAYLOR, R.J. \& C.E.S. TAYLOR. 1994. An annotated list of the ferns, fern allies, gymnosperms, and flowering plants of Oklahoma. 3rd Ed. Biology Dept. Herbarium, Southeastern Oklahoma State Univ., Durant, U.S.A.

Thomas, R.D. \& C.M. Allen. 1993. Atlas of the vascular flora of Louisiana. Vol. 1. Louisiana Dept. Wildlife \& Fisheries and The Nature Conservancy, Louisiana Field Office, Baton Rouge, U.S.A.

Turland, N. J., J.H. Wiersema, F.R. Barrie, W. Greuter, D.L. Hawksworth, P.S. Herendeen, S. Knapp, W.-H. Kusber, D.-Z. Li, K. Marhold, T.W. MAY, J. McNeill, A.M. MonRo, J. Prado, M.J. Price, \& G.F. SMITH, EDS. 2018. International code of nomenclature for algae, fungi, and plants (Shenzhen Code) adopted by the Nineteenth International Botanical Congress Shenzhen, China, July 2017. Regnum Veg. 159.

WAGneR, W.L., P.C. Hoch, \& P.H. RAven. 2007. Revised classification of the Onagraceae. Syst. Bot. Monogr. 83:1-240.

WALTER, T. 1788. Flora Caroliniana. London, U.K.

WARD, D.B. 2007a. The Thomas Walter herbarium is not the herbarium of Thomas Walter. Taxon 56:917-926.

WARD, D.B. 2007b. Thomas Walter typification project, II. The known Walter types. J. Bot. Res. Inst. Texas 1:407-423.

WARD, D.B. 2017. Thomas Walter and his plants. New York Botanical Garden Press, Bronx, NY, U.S.A.

WEAKLEY, A.S. 2015. Flora of the southern and mid-Atlantic states, working draft of May 2015. University of North Carolina Herbarium, North Carolina Botanical Garden, Chapel Hill, U.S.A. http://www.herbarium.unc.edu/flora.htm.

WEAKLEY, A.S. 2018. Flora of the southern and mid-Atlantic states. University of North Carolina Herbarium, North Carolina Botanical Garden, Chapel Hill, U.S.A.

Weakley, A.S., D.B. Poindexter, R.J. LeBlond, B.A. Sorrie, C.H. Karlsson, P.J. Williams, E.L. Bridges, S.L. Orzell, B.R. Keener, A. Weeks, R.D. Noyes, M. Flores-Cruz, J.T. Diggs, G.D. Gann, \& A.J. Floden. 2017. New combinations, rank changes, and nomenclatural and taxonomic comments in the vascular flora of the southeastern United States. II. J. Bot. Res. Inst. Texas 11:291-325.

WEl, Z.X. 1995. Pollen morphology of Trillium. Acta Bot. Yunnanica 17:317-324. 